\title{
On controllability methods for the Helmholtz equation
}

\author{
M. J. Grote and J. H. Tang
}

Departement Mathematik und Informatik

Preprint No. 2018-06

May 2018

Universität Basel

CH-4051 Basel

www.math.unibas.ch 


\title{
ON CONTROLLABILITY METHODS FOR THE HELMHOLTZ EQUATION
}

\author{
MARCUS J. GROTE AND JET HOE TANG
}

\begin{abstract}
When the Helmholtz equation is discretized by standard finite difference or finite element methods, the resulting linear system is highly indefinite and thus notoriously difficult to solve, in fact increasingly so at higher frequency. The exact controllability approach [1] instead reformulates the problem in the time domain and seeks the time-harmonic solution of the corresponding wave equation. By iteratively reducing the mismatch between the solution at initial time and after one period, the controllability method greatly speeds up the convergence to the time-harmonic asymptotic limit. Moreover, each conjugate gradient iteration solely relies on standard numerical algorithms, which are inherently parallel and robust against higher frequencies. The original energy functional used to penalize the departure from periodicity is strictly convex only for sound-soft scattering problems. To extend the controllability approach to general boundaryvalue problems governed by the Helmholtz equation, new penalty functionals are proposed, which are numerically efficient. Numerical experiments for wave scattering from sound-soft and sound-hard obstacles, inclusions, but also for wave propagation in closed wave guides illustrate the usefulness of the resulting controllability methods.
\end{abstract}

\section{INTRODUCTION}

For time-harmonic wave phenomena, that is, for wave phenomena governed by a single time frequency $\omega$, the efficient numerical solution of the Helmholtz equation is of fundamental importance. Although the Helmholtz equation merely results from an apparently benign diagonal shift from an elliptic coercive operator, that resemblance is deceiving. Indeed, the performance of standard numerical methods for Laplace/Poisson-type problems quickly deteriorates when applied to the Helmholtz equation, in fact increasingly so at higher frequency, which thus remains notoriously difficult to solve [2]. Despite the recent development of various preconditioners to accelerate the convergence of standard iterative methods [3-7], the numerical solution of the Helmholtz equation in three-dimensional heterogeneous media remains a formidable challenge.

To circumvent those difficulties, Bristeau, Glowinski and Périaux proposed twenty years ago an alternative method using exact controllability $[1,8,9]$. Instead of solving the Helmholtz equation directly in the frequency domain, the controllability method

Date: May 2, 2018.

Key words and phrases. Helmholtz equation; time-harmonic waves; time-periodic waves; scattering; exact controllability; finite element method; local time-stepping. 
(CM) transforms the problem back to the time domain, where it seeks a periodic solution $y(\cdot, t)$ with (known) period $T=2 \pi / \omega$ of the corresponding time-dependent wave equation. The unknown initial conditions, $v_{0}=y(\cdot, 0)$ and $v_{1}=y_{t}(\cdot, 0)$, that yield the desired periodic solution are then determined by minimizing a convex cost functional, $J_{1}\left(v_{0}, v_{1}\right)$, which penalizes the departure from periodicity. Akin to a shooting method, the controllability approach iteratively solves the least-squares optimization problem with a standard conjugate gradient (CG) iteration [10]. Each CG iteration then requires the solution of a forward and a backward wave equation together with the solution of a symmetric and positive definite linear system independent of $\omega$, both easily solved using standard numerical methods. Hence, the CM-CG approach solely relies on standard numerical algorithms, which are not only robust with respect to $\omega$ but also easy to parallelize. In [11], Bardos and Rauch proved the uniqueness of the minimizer for sound-soft exterior Helmholtz problems. They also proposed an alternative functional, $J_{\infty}\left(v_{0}, v_{1}\right)$, which is unconditionally coercive even for trapping obstacles. Later Koyama proved convergence of the CM-CG method based on $J_{1}$ for sound-soft wave scattering from a disk [12].

The CM-CG method in $[1,8]$ relied on a piecewise linear finite element (FE) discretization in space and the second-order leapfrog scheme in time. Low-order FE discretizations, however, are notoriously prone to the pollution effect [13]. Moreover, local mesh refinement imposes a severe CFL stability constraint on explicit time integration, as the maximal time-step is dictated by the smallest element in the mesh. Recently, Heikkola et al. [14,15] combined higher-order $\mathcal{P}^{3}$ spectral FE with the classical fourth-order Runge-Kutta (RK) method to mitigate the pollution effect. They also used an algebraic multigrid preconditioner to remove mesh dependence of the convergence at fixed frequency.

So far the controllability method has always been applied to sound-soft scattering problems $[1,8,14,15]$, where both Dirichlet and impedance conditions are imposed at the boundary. However, for wave scattering from sound-hard obstacles, inclusions, or othe wave phenomena in physically bounded domains, the original CM-CG method will generally fail because the minimizer of $J_{1}$ is no longer unique. Although the alternative functional $J_{\infty}$ from [11] in fact restores uniqueness of the minimizer in all those problem settings, it also requires storing the entire history of the solution of the wave equation, which may be prohibitive for large-scale problems.

Here, we address those difficulties and show how to efficiently extend the CM-CG method to general boundary value problems governed by the Helmholtz equation. In Section 2, we consider the Helmholtz equation in a general setting and reformulate the boundary value problem in the time domain. Then, we recall the original CMCG method based on $J_{1}$ for sound-soft scattering problems $[1,8]$ and show that it does not yield the expected (unique) solution for scattering problems with soundhard obstacles or inclusions. To guarantee that the CM-CG method converges to the correct solution of the Helmholtz equation at little extra cost, we either post-process it via a compatibility condition or propose an alternative functional $J_{m}, m \geqslant 2$. In Section 3 , we show that $J_{3}$ always yields the (unique) time-harmonic solution of the Helmholtz equation, even in a physically bounded domain with Dirichlet or Neumann conditions only. Finally, in Section 4, we present numerical experiments for various 


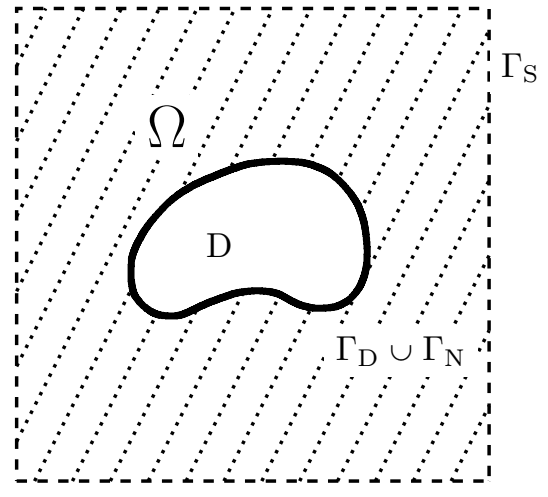

(a) unbounded domain with obstacle $D$, truncated by an artificial boundary $\Gamma_{S}$.

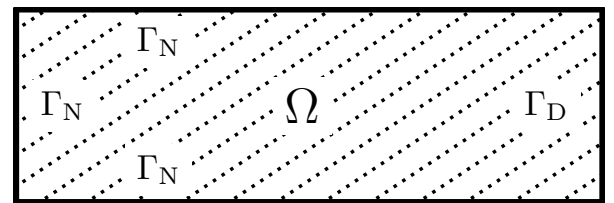

(b) physically bounded domain with Dirichlet and Neumann conditions.

Figure 1. Typical bounded computational domain $\Omega$

scatterers, but also for wave propagation in physically bounded domains to illustrate the usefulness of these improvements. In particular, we show how recent explicit local time-stepping methods [16] overcome the bottleneck due to local mesh refinement in the time integration of the forward and backward wave equations.

\section{Controllability Method for Scattering Problems}

We consider the Helmholtz equation in a bounded computational domain $\Omega \subset \mathbb{R}^{d}$, $d \leqslant 3$, whose boundary $\Gamma$ generally consists of physical and artificial boundaries, see Figure 1. More precisely, we assume that $\Gamma$ consists of three disjoint components, $\Gamma=\Gamma_{D} \cup \Gamma_{N} \cup \Gamma_{S}$, each possibly empty, where we impose a Dirichlet, Neumann and impedance or Sommerfeld-like absorbing boundary condition, respectively. Hence, the wave field $u$ satisfies the Helmholtz equation

$$
\begin{aligned}
-\nabla \cdot\left(c^{2}(x) \nabla u(x)\right)-\omega^{2} u(x) & =f(x), & & x \in \Omega, \\
\frac{\partial u(x)}{\partial \nu}-i \frac{\omega}{c_{0}} u(x) & =g_{S}(x), & & x \in \Gamma_{S}, \\
\frac{\partial u(x)}{\partial \nu} & =g_{N}(x), & & x \in \Gamma_{N}, \\
u(x) & =g_{D}(x), & & x \in \Gamma_{D} .
\end{aligned}
$$

Here $\omega>0$ denotes the frequency and $c(x) \geqslant c_{\min }>0$ the propagation speed of the medium, which is assumed constant $c(x) \equiv c_{0}$ in the vicinity of $\Gamma_{S}$. Moreover, $\nu$ denotes the unit outward normal while $f, g_{N}, g_{S}$ and $g_{D}$ are known and may vanish.

For standard sound-soft or sound-hard scattering problems, for instance, $\Gamma_{S}$ corresponds to an artificial boundary, which truncates the unbounded exterior whereas the remaining components, $\Gamma_{D} \cup \Gamma_{N}$, correspond to the physical boundary of the obstacle; in the absence of any obstacle, $\Gamma_{D}=\Gamma_{N}=\varnothing$. Other configurations, such 
as open wave guides or physically bounded domains are also included in our formulation. Hence, the setting in (2.1) is rather general and encompasses most common applications.

For constant $c(x) \equiv c_{0}>0$, it is well-known that (2.1) has a unique solution $u \in H^{1}(\Omega)$ if $\left|\Gamma_{S}\right|>0$ or if $\omega^{2}$ is not an eigenvalue of the principal elliptic part [17]. Based on the unique continuation principle [18,19], Graham and Sauter recently extended this result to the variable coefficient case [20]. Henceforth we shall assume for any particular choice of $\Gamma_{D}, \Gamma_{N}, \Gamma_{S}, c(x)$ and $\omega$ that the boundary value problem (2.1) has a unique solution $u \in H^{1}(\Omega)$.

Instead of solving the Helmholtz equation directly in the frequency domain, we now reformulate (2.1) in the time domain. Hence, the corresponding time-harmonic wave field, $\operatorname{Re}\left\{u(x) \mathrm{e}^{-i \omega t}\right\}$, satisfies the (real-valued) time-dependent wave equation

$$
\begin{aligned}
y_{t t}(x, t)-\nabla \cdot\left(c^{2}(x) \nabla y(x, t)\right) & =\operatorname{Re}\left\{f(x) \mathrm{e}^{-i \omega t}\right\}, & & x \in \Omega, t>0, \\
\frac{\partial y(x, t)}{\partial \nu}+\frac{1}{c_{0}} \frac{\partial y(x, t)}{\partial t} & =\operatorname{Re}\left\{g_{S}(x) \mathrm{e}^{-i \omega t}\right\}, & & x \in \Gamma_{S}, t>0, \\
\frac{\partial y(x, t)}{\partial \nu} & =\operatorname{Re}\left\{g_{N}(x) \mathrm{e}^{-i \omega t}\right\}, & & x \in \Gamma_{N}, t>0, \\
y(x, t) & =\operatorname{Re}\left\{g_{D}(x) \mathrm{e}^{-i \omega t}\right\}, & & x \in \Gamma_{D}, t>0, \\
y(x, 0) & =v_{0}, & & x \in \Omega, \\
y_{t}(x, 0) & =v_{1}, & & x \in \Omega,
\end{aligned}
$$

for the (unknown) initial values $v_{0}=\operatorname{Re}\{u\}$ and $v_{1}=\omega \operatorname{Im}\{u\}$. Again it is wellknown that $(2.2)$ is well-posed and has a unique solution $y \in C^{0}\left(0, T ; H^{1}(\Omega)\right) \cap$ $C^{1}\left(0, T ; L^{2}(\Omega)\right)$ for given $v_{0} \in H^{1}(\Omega)$ and $v_{1} \in L^{2}(\Omega)[21,22]$. Note that for the desired time-harmonic wave field, $\operatorname{Re}\left\{u(x) \mathrm{e}^{-i \omega t}\right\}$, not only the solution but also its time derivative lie in $H^{1}(\Omega)$ for all time.

For sound-soft scattering problems where $\left|\Gamma_{D}\right|>0$ and $\left|\Gamma_{S}\right|>0$, Bristeau et al. $[8,9]$ proposed to determine the solution $u(x)$ of $(2.1)$ by computing a time-periodic solution $y(x, t)$ of $(2.2)$ with period $T=2 \pi / \omega$. Its initial values $v_{0}, v_{1}$, once known, then determine the solution $u$ of the original Helmholtz equation (2.1) as

$$
u=v_{0}+\frac{i}{\omega} v_{1}, \quad v_{0}, v_{1} \in H^{1}(\Omega) .
$$

To determine $v_{0}$ and $v_{1}$, the problem is reformulated as a least-squares optimization problem $[1,8]$ over $H^{1}(\Omega) \times L^{2}(\Omega)$ for the quadratic cost functional

$$
J_{1}\left(v_{0}, v_{1}\right)=\frac{1}{2} \int_{\Omega}\left|c(x) \nabla\left(y(x, T)-v_{0}(x)\right)\right|^{2} d x+\frac{1}{2} \int_{\Omega}\left(y_{t}(x, T)-v_{1}(x)\right)^{2} d x
$$

where $y$ satisfies (2.2). The functional $J_{1}$ measures in the energy norm the mismatch between the solution of $(2.2)$ at the initial time and after one period. It is nonnegative and convex, while $J_{1}\left(v_{0}, v_{1}\right)=0$ if, and only if, $\nabla y$ and $y_{t}$ are $T$-periodic in time for the initial values $\left(v_{0}, v_{1}\right)$; in particular, $J_{1}\left(v_{0}, v_{1}\right)=0$ for $v_{0}=\operatorname{Re}\{u\}$ and $v_{1}=\omega \operatorname{Im}\{u\}$. If $J_{1}$ has a unique minimizer, $y(x, t)$ necessarily coincides with $\operatorname{Re}\left\{u(x) \mathrm{e}^{-i \omega t}\right\}$ and hence $v_{0}, v_{1} \in H^{1}(\Omega)$. 
For sound-soft scattering problems, where we impose both Dirichlet and impedance boundary conditions in (2.1), the functional $J_{1}$ in fact has a unique minimizer [11], which therefore coincides with the (unique) time-harmonic solution $\operatorname{Re}\left\{u(x) \mathrm{e}^{-i \omega t}\right\}$ of (2.2). In general, however, a minimizer of $J_{1}$ is neither unique nor does it necessarily yield the (unique) time-harmonic solution, as shown in the following theorem.

Theorem 1. Let $u \in H^{1}(\Omega)$ be the unique solution of the Helmholtz equation (2.1) and $y \in C^{0}\left([0, T] ; H^{1}(\Omega)\right) \cap C^{1}\left([0, T] ; L^{2}(\Omega)\right)$ be a (real-valued) solution of the wave equation (2.2) with initial conditions $\left(v_{0}, v_{1}\right) \in H^{1}(\Omega) \times L^{2}(\Omega)$. If $\nabla y$ and $y_{t}$ are time periodic with period $T=2 \pi / \omega$, then $y$ admits the Fourier series expansion

$$
(y(\cdot, t), \varphi)=\left(\operatorname{Re}\left\{u \mathrm{e}^{-i \omega t}\right\}, \varphi\right)+(\lambda+\eta t, \varphi)+\sum_{\ell=1}^{\infty}\left(\alpha_{\ell} \cos (\omega \ell t)+\beta_{\ell} \sin (\omega \ell t), \varphi\right)
$$

for any $\varphi \in H_{D}^{1}$, where the constants $\lambda, \eta \in \mathbb{R}$ and the eigenfunctions $\alpha_{\ell}, \beta_{\ell} \in H_{D}^{1}$, $\ell \geqslant 1$, satisfy

$$
\begin{aligned}
-\nabla \cdot\left(c^{2}(x) \nabla \alpha_{\ell}(x)\right) & =(\omega \ell)^{2} \alpha_{\ell}(x), & & x \in \Omega,, \\
\alpha_{\ell}(x) & =0, & & x \in \Gamma_{D}, \\
\frac{\partial \alpha_{\ell}(x)}{\partial \nu} & =-\frac{\omega \ell}{c_{0}} \beta_{\ell}(x), & & x \in \Gamma_{S}, \\
\frac{\partial \alpha_{\ell}(x)}{\partial \nu} & =0, & & x \in \Gamma_{N}, \\
-\nabla \cdot\left(c^{2}(x) \nabla \beta_{\ell}(x)\right) & =(\omega \ell)^{2} \beta_{\ell}(x), & & x \in \Omega, \\
\beta_{\ell}(x) & =0, & & x \in \Gamma_{D}, \\
\frac{\partial \beta_{\ell}(x)}{\partial \nu} & =\frac{\omega \ell}{c_{0}} \alpha_{\ell}(x), & & x \in \Gamma_{S}, \\
\frac{\partial \beta_{\ell}(x)}{\partial \nu} & =0, & & x \in \Gamma_{N} .
\end{aligned}
$$

Let $v=v_{0}+(i / \omega) v_{1}$. Then $v$ satisfies

$$
(v, \varphi)=(u, \varphi)+\left(\lambda+\frac{i}{\omega} \eta, \varphi\right)+\sum_{\ell=1}^{\infty}\left(\alpha_{\ell}+i \ell \beta_{\ell}, \varphi\right) \quad \forall \varphi \in H_{D}^{1} .
$$

Furthermore, if $\left|\Gamma_{D} \cup \Gamma_{S}\right|>0$, then $\eta=0$ and if $\left|\Gamma_{D}\right|>0$, then $\lambda=\eta=0$.

Here $H_{D}^{1}=\left\{\tilde{v} \in H^{1}(\Omega):\left.\tilde{v}\right|_{\Gamma_{D}}=0\right\}$ and $(\cdot, \cdot)$ denotes the standard inner product on $L^{2}(\Omega)$

From (2.8) we conclude that $u=v_{0}+(i / \omega) v_{1}$ precisely when $\lambda=\eta=0$ and all $\alpha_{\ell}, \beta_{\ell}, \ell \geqslant 1$, vanish identically. For later reference, we note that the two realvalued eigenvalue problems (2.6)-(2.7) can be combined into a single complex-valued eigenvalue problem as follows.

Remark 1. Let

$$
\gamma_{\ell}=\alpha_{\ell}+i \beta_{\ell}, \quad \gamma_{-\ell}=\bar{\gamma}_{\ell}, \quad \ell=1,2, \ldots
$$


Then $\alpha_{\ell}$ and $\beta_{\ell}$ solve (2.6)-(2.7) if, and only if, $\gamma_{\ell}$ solves the homogeneous Helmholtz equation with frequency $\omega \ell$ :

$$
\begin{aligned}
-\nabla \cdot\left(c^{2}(x) \nabla \gamma_{\ell}(x)\right)-(\omega \ell)^{2} \gamma_{\ell}(x) & =0, & & x \in \Omega, \\
\frac{\partial \gamma_{\ell}(x)}{\partial \nu}-i \frac{\omega \ell}{c_{0}} \gamma_{\ell}(x) & =0, & & x \in \Gamma_{S}, \\
\frac{\partial \gamma_{\ell}(x)}{\partial \nu} & =0, & & x \in \Gamma_{N}, \\
\gamma_{\ell}(x) & =0, & & x \in \Gamma_{D} .
\end{aligned}
$$

Hence, $\gamma_{\ell}$, or equivalently $\alpha_{\ell}$ and $\beta_{\ell}$, vanish precisely when (2.10) only admits the trivial solution for all $\ell \neq 0$. In particular, if $\left|\Gamma_{S}\right|>0$, then $\gamma_{\ell}=0, \ell \neq 0$.

Proof. For the proof, we distinguish between (i) $\left|\Gamma_{D} \cup \Gamma_{S}\right|>0$ and (ii) $\Gamma_{D} \cup \Gamma_{S}=\varnothing$.

(i) For $\left|\Gamma_{D} \cup \Gamma_{S}\right|>0$, let $z$ denote the difference between $y(x, t)$ and the timeharmonic solution:

$$
z(x, t)=y(x, t)-\operatorname{Re}\left\{u(x) \mathrm{e}^{-i \omega t}\right\}
$$

It is easy to verify that $z$ satisfies

$$
\begin{aligned}
z_{t t}(x, t)-\nabla \cdot\left(c^{2}(x) \nabla z(x, t)\right) & =0, & & (x, t) \in \Omega \times(0, T), \\
z(x, t) & =0, & & (x, t) \in \Gamma_{D} \times(0, T), \\
\frac{\partial z(x, t)}{\partial \nu}+\frac{1}{c_{0}} \frac{\partial z(x, t)}{\partial t} & =0, & & (x, t) \in \Gamma_{S} \times(0, T),
\end{aligned}
$$

while both $\nabla z$ and $z_{t}$ are $T$-periodic. Hence, $\nabla z(\cdot, T)-\nabla z(\cdot, 0)=0$ throughout $\Omega$ and $\eta(x)=z(x, T)-z(x, 0)$ is constant a.e. in $\Omega$.

If $\left|\Gamma_{D}\right|>0, \eta$ vanishes on $\Gamma_{D}$ and hence $\eta=0$ everywhere in $\Omega$. Otherwise $\Gamma_{D}=\varnothing$ and thus $\left|\Gamma_{S}\right|>0$. By integrating (2.12a) and using Green's formula together with the homogeneous boundary conditions on $\Gamma_{N}$ and $\Gamma_{S}$, we obtain

$$
0=\int_{0}^{T} \int_{\Omega} z_{t t}-\nabla \cdot\left(c^{2} \nabla z\right) d x d t=\left.\int_{\Omega} z_{t}\right|_{0} ^{T} d x+\left.\int_{\Gamma_{S}} c_{0} z\right|_{0} ^{T} d s=\eta c_{0}\left|\Gamma_{S}\right|,
$$

where we have used $(2.12 \mathrm{c})$ and the $T$-periodicity of $z_{t}$. Since $c_{0}>0$ and $\left|\Gamma_{S}\right|>0$, $\eta$ must equal zero. Therefore, $z$ is $T$-periodic and for any $\varphi \in H_{D}^{1}$, the function $(z(\cdot, t), \varphi):[0, T] \rightarrow \mathbb{R}$ is also $T$-periodic and continuous (Theorem 2, §5.9.2 in [21]). Thus, we can expand it in Fourier series as

$$
(z(\cdot, t), \varphi)=\frac{\tilde{\alpha}_{0}}{2}+\sum_{\ell=1}^{\infty}\left(\tilde{\alpha}_{\ell} \cos (\omega \ell t)+\tilde{\beta}_{\ell} \sin (\omega \ell t)\right), \quad 0 \leqslant t \leqslant T,
$$

with Fourier coefficients

$$
\tilde{\alpha}_{\ell}=\frac{2}{T} \int_{0}^{T}(z(\cdot, t), \varphi) \cos (\omega \ell t) d t, \quad \tilde{\beta}_{\ell}=\frac{2}{T} \int_{0}^{T}(z(\cdot, t), \varphi) \sin (\omega \ell t) d t .
$$


Now let

$$
\begin{aligned}
& \alpha_{\ell}(x)=\frac{2}{T} \int_{0}^{T} z(x, t) \cos (\omega \ell t) d t, \ell \geqslant 0, \\
& \beta_{\ell}(x)=\frac{2}{T} \int_{0}^{T} z(x, t) \sin (\omega \ell t) d t, \quad \ell \geqslant 1 .
\end{aligned}
$$

Then $\tilde{\alpha}_{\ell}=\left(\alpha_{\ell}, \varphi\right)$ and $\tilde{\beta}_{\ell}=\left(\beta_{\ell}, \varphi\right)$.

Next, we verify that $\alpha_{\ell}$ and $\beta_{\ell}$ solve the eigenvalue problems (2.6) and (2.7) respectively. First, we replace $\alpha_{\ell}$ on the left side of (2.6) by its definition (2.14a), use (2.12a), and integrate by parts twice with respect to time the resulting expression to obtain

$$
\begin{aligned}
-\nabla \cdot\left(c^{2}(x) \nabla \alpha_{\ell}(x)\right)= & -\frac{2}{T} \int_{0}^{T} \nabla \cdot\left(c^{2}(x) \nabla z(x, t)\right) \cos (\omega \ell t) d t \\
= & -\frac{2}{T} \int_{0}^{T} z_{t t}(x, t) \cos (\omega \ell t) d t \\
= & (\omega \ell)^{2} \frac{2}{T} \int_{0}^{T} z(x, t) \cos (\omega \ell t) d t \\
& -\left.\frac{2 \omega \ell}{T} z(x, t) \sin (\omega \ell t)\right|_{0} ^{T}-\left.\frac{2}{T} z_{t}(x, t) \cos (\omega \ell t)\right|_{0} ^{T}, \quad x \in \Omega .
\end{aligned}
$$

Since both $z$ and $z_{t}$ are $T$-periodic, the last two terms vanish, which yields

$$
-\nabla \cdot\left(c^{2}(x) \nabla \alpha_{\ell}(x)\right)=(\omega \ell)^{2} \frac{2}{T} \int_{0}^{T} z(x, t) \cos (\omega \ell t) d t=(\omega \ell)^{2} \alpha_{\ell}(x), \quad x \in \Omega,
$$

by definition (2.14a) of $\alpha_{\ell}$.

We still need to verify that $\alpha_{\ell}$ also satisfies the boundary conditions $(2.6 \mathrm{~b})$ and (2.6c). As $z(\cdot, t)$ vanishes on $\Gamma_{D}$, so does $\alpha_{\ell}$ since

$$
\alpha_{\ell}(x)=\frac{2}{T} \int_{0}^{T} z(x, t) \cos (\omega \ell t) d t=0, \quad x \in \Gamma_{D} .
$$

To verify that $\alpha_{\ell}$ satisfies (2.6c) on $\Gamma_{S}$, we start from (2.14a) and again use integration by parts in time, the periodicity of $z$, and (2.12c) to obtain

$$
\begin{aligned}
\frac{\partial \alpha_{\ell}(x)}{\partial \nu} & =\frac{2}{T} \int_{0}^{T} \frac{\partial z(x, t)}{\partial \nu} \cos (\omega \ell t) d t=-\frac{2}{T c_{0}} \int_{0}^{T} \frac{\partial z(x, t)}{\partial t} \cos (\omega \ell t) d t \\
& =-\frac{2 \omega \ell}{T c_{0}} \int_{0}^{T} z(x, t) \sin (\omega \ell t) d t-\left.\frac{2}{T c_{0}} z(x, t) \cos (\omega \ell t)\right|_{0} ^{T}=-\frac{\omega \ell}{c_{0}} \beta_{\ell}(x), \quad x \in \Gamma_{S},
\end{aligned}
$$

by definition (2.14b) of $\beta_{\ell}$. Hence, $\alpha_{\ell}$ indeed satisfies (2.6) for $\ell \geqslant 0$.

In particular, for $\ell=0$ equation (2.6) yields

$$
\begin{aligned}
-\nabla \cdot\left(c^{2} \nabla \alpha_{0}\right) & =0 & & \text { in } \Omega, \\
\alpha_{0} & =0 & & \text { on } \Gamma_{D}, \\
\frac{\partial \alpha_{0}}{\partial \nu} & =0 & & \text { on } \Gamma_{S} .
\end{aligned}
$$


Multiplication of (2.15) by $\alpha_{0}$ and integration by parts over $\Omega$ then implies

$$
\left\|c \nabla \alpha_{0}\right\|_{L^{2}(\Omega)}^{2}=-\left(\nabla \cdot\left(c^{2} \nabla \alpha_{0}\right), \alpha_{0}\right)_{L^{2}(\Omega)}=0 .
$$

Therefore, $\alpha_{0}$ is constant throughout $\Omega$ and we define $\lambda=\alpha_{0} / 2$. Similar calculations show that $\beta_{\ell}$ satisfies (2.7) for any $\ell \geqslant 1$.

Next, we use (2.13) in (2.11) and rearrange terms to obtain

$(y(\cdot, t), \varphi)=\left(\operatorname{Re}\left\{u \mathrm{e}^{-i \omega t}\right\}, \varphi\right)+(\lambda, \varphi)+\sum_{\ell=1}^{\infty}\left(\alpha_{\ell} \cos (\omega \ell t)+\beta_{\ell} \sin (\omega \ell t), \varphi\right), \quad \forall \varphi \in H_{D}^{1}$,

which corresponds to (2.8) with $\eta=0$.

In particular, (2.5) implies that

$$
\begin{aligned}
& \left(v_{0}, \varphi\right)=(y(\cdot, 0), \varphi)=(\operatorname{Re}\{u\}, \varphi)+(\lambda, \varphi)+\sum_{\ell=1}^{\infty}\left(\alpha_{\ell}, \varphi\right), \\
& \left(v_{1}, \varphi\right)=\left(y_{t}(\cdot, 0), \varphi\right)=\omega(\operatorname{Im}\{u\}, \varphi)+\omega \sum_{\ell=1}^{\infty}\left(\ell \beta_{\ell}, \varphi\right),
\end{aligned}
$$

for any $\varphi \in H_{D}^{1}$. By combining (2.16) and (2.17) we thus obtain

$$
(v, \varphi)=\left(v_{0}+\frac{i}{\omega} v_{1}, \varphi\right)=(u, \varphi)+(\lambda, \varphi)+\sum_{\ell=1}^{\infty}\left(\alpha_{\ell}+i \ell \beta_{\ell}, \varphi\right)
$$

which corresponds to (2.8) with $\eta=0$.

(ii) For $\Gamma_{D}=\Gamma_{S}=\varnothing$, that is in a situation of pure Neumann boundary conditions, $y$ is not $T$-periodic in general and hence

$$
\eta(x)=\frac{1}{T}(y(x, T)-y(x, 0))
$$

is no longer zero. However, $\nabla \eta(x) \equiv 0$ by assumption and thus $\eta$ is constant a.e. in $\Omega$. Now, let

Clearly, $z$ satisfies

$$
z(x, t)=y(x, t)-\operatorname{Re}\left\{u(x) \mathrm{e}^{-i \omega t}\right\}-\eta t .
$$

$$
\begin{aligned}
z_{t t}(x, t)-\nabla \cdot\left(c^{2}(x) \nabla z(x, t)\right) & =0, & & (x, t) \in \Omega \times[0, T], \\
\frac{\partial z(x, t)}{\partial \nu} & =0, & & (x, t) \in \Gamma_{N} \times[0, T],
\end{aligned}
$$

and is $T$-periodic by definition of $\eta$. Therefore, $z$ again admits a Fourier series expansion with coefficients $\alpha_{\ell}$ and $\beta_{\ell}$ which satisfy (2.6) and (2.7), respectively. A similar argument as in case (i) concludes the proof.

From Theorem 1, we conclude that the initial values $v_{0}$ and $v_{1}$ of a time-periodic solution are in general not unique whenever $\lambda, \eta, \alpha_{\ell}$ or $\beta_{\ell}$ is not identically zero. For sound-soft scattering problems where $\left|\Gamma_{S}\right|>0$ and $\left|\Gamma_{D}\right|>0$, however, the constants $\lambda, \eta$ and the eigenfunctions all vanish. Then, $\left(v_{0}, v_{1}\right)$ is the unique minimizer of $J_{1}$ in (2.4) which yields the solution $u$ of (2.1) through (2.3). We summarize this result in the following corollary - see also [12]. 
Corollary 1. Let $u \in H^{1}(\Omega)$ be the unique solution of (2.1) with $\left|\Gamma_{S}\right|>0,\left|\Gamma_{D}\right|>0$ and $y \in C^{0}\left([0, T] ; H^{1}(\Omega)\right) \cap C^{1}\left([0, T] ; L^{2}(\Omega)\right)$ be a (real-valued) solution of (2.2) with initial conditions $\left(v_{0}, v_{1}\right) \in H^{1}(\Omega) \times L^{2}(\Omega)$. If $\nabla y$ and $y_{t}$ are time periodic with period $T=2 \pi / \omega$, then

$$
u=v_{0}+\frac{i}{\omega} v_{1}
$$

Proof. From Theorem 1 we know that $u$ satisfies the Fourier expansion (2.8) with $\lambda=\eta=0$. Since each eigenvalue problem (2.10) corresponds to the Helmholtz equation (2.1) with frequency $\omega \ell$ and $f=g_{S}=g_{D}=g_{N} \equiv 0, \ell \neq 0$, which has only the trivial solution, we conclude that $\alpha_{\ell}=\beta_{\ell}=0, \ell \geqslant 1$. Therefore (2.3) holds with $\alpha_{\ell}=\beta_{\ell}=0, \ell \geqslant 1$, and $\lambda=\eta=0$.

2.1. Sound-Soft Scattering. Let $u$ be the (unique) solution of the Helmholtz equation (2.1) with $\left|\Gamma_{D}\right|>0$ and $\left|\Gamma_{S}\right|>0$, as in a typical sound-soft scattering problem where $\Gamma_{D}$ corresponds to the boundary of the obstacle and $\Gamma_{S}$ to the exterior artificial boundary. By Corollary 1 solving (2.1) is then equivalent to minimizing over $H^{1}(\Omega) \times L^{2}(\Omega)$ the functional $J_{1}$ defined by (2.4). Since $J_{1}$ is quadratic, a natural choice to minimize $J_{1}$ is the conjugate gradient (CG) method $[1,8]$, which requires the Fréchet derivative of $J_{1}$ at $v=\left(v_{0}, v_{1}\right)$ for a perturbation $\delta v=\left(\delta v_{0}, \delta v_{1}\right)$ :

$$
\begin{aligned}
\left\langle J_{1}^{\prime}(v), \delta v\right\rangle= & -\int_{\Omega} c^{2}(x) \nabla\left(y(x, T)-v_{0}(x)\right) \nabla \delta v_{0}(x) d x \\
& -\int_{\Omega}\left(y_{t}(x, T)-v_{1}(x)\right) \delta v_{1}(x) d x \\
& +\int_{\Omega}\left(p(x, 0) \delta v_{1}(x)-p_{t}(x, 0) \delta v_{0}(x)\right) d x+c_{0} \int_{\Gamma_{S}} p(x, 0) \delta v_{0}(x) d s
\end{aligned}
$$

where $\langle\cdot, \cdot\rangle$ denotes the standard duality pairing and $p$ is the solution of the adjoint (backward) wave equation,

$$
\begin{aligned}
p_{t t}(x, t)-\nabla \cdot\left(c^{2}(x) \nabla p(x, t)\right) & =0, & & x \in \Omega, t>0, \\
\frac{\partial p(x, t)}{\partial \nu}-\frac{1}{c_{0}} p_{t}(x, t) & =0, & & x \in \Gamma_{S}, t>0 \\
\frac{\partial p(x, t)}{\partial \nu} & =0, & & x \in \Gamma_{N}, t>0, \\
p(x, t) & =0, & & x \in \Gamma_{D}, t>0, \\
p(x, T) & =p_{0}(x), & & x \in \Omega, \\
p_{t}(x, T) & =p_{1}(x), & & x \in \Omega,
\end{aligned}
$$

and the initial conditions satisfy for any $w \in H_{D}^{1}(\Omega)$

$$
\begin{aligned}
p_{0}(x) & =y_{t}(x, T)-v_{1}(x), \quad x \in \Omega \\
\int_{\Omega} p_{1}(x) w(x) d x & =c_{0} \int_{\Gamma_{S}} p_{0}(x) w(x) d s \\
& -\int_{\Omega} c^{2}(x) \nabla\left(y(x, T)-v_{0}(x)\right) \nabla w(x) d x .
\end{aligned}
$$


The derivation of (2.19) and (2.20) and the CM-CG Algorithm can be found in $[1,8]$. Hence in each CG iteration, we compute $J_{1}^{\prime}(v)$ by solving the forward and backward (adjoint) wave equations $(2.2)$ and $(2.20)$ over one period $[0, T]$. If the time-step $\Delta t$ until $T=2 \pi / \omega$ is chosen inversely proportional to the frequency $\omega$, the number of time-steps over one period remains independent of $\omega$. Moreover, each CG iteration requires an explicit (Riesz) representer $\tilde{g}=\left(\tilde{g}_{0}, \tilde{g}_{1}\right) \in H_{D}^{1}(\Omega) \times L^{2}(\Omega)$ of the gradient $g=\left(g_{0}, g_{1}\right)=J_{1}^{\prime}(v)$ which is determined by solving the symmetric and coercive elliptic problem $[8,23]$ :

$$
\begin{aligned}
\int_{\Omega} c^{2}(x) \nabla \tilde{g}_{0}(x) \nabla \varphi(x) d x & =\int_{\Omega} g_{0}(x) \varphi(x) d x \\
& =\int_{\Omega} c^{2}(x) \nabla\left(v_{0}(x)-y(x, T)\right) \nabla \varphi(x)-p_{t}(x, 0) \varphi(x) d x \\
& +c_{0} \int_{\Gamma_{S}} p(x, 0) \varphi(x) d s, \quad \forall \varphi \in H_{D}^{1}, \\
\tilde{g}_{1}(x) & =g_{1}(x)=v_{1}(x)-y_{t}(x, T)+p(x, 0), \quad x \in \Omega .
\end{aligned}
$$

For the sake of future reference, we list the CM-CG Algorithm from $[1,8]$ below.

\section{CM-CG Algorithm.}

(1) Initialize $v^{(0)}=\left(v_{0}^{(0)}, v_{1}^{(0)}\right)$ (initial guess).

(2) Solve the forward and the backward wave equations (2.2) and (2.20) to determine the gradient of $J_{1}, g^{(0)}=J_{1}^{\prime}\left(v^{(0)}\right)$, defined by (2.19).

(3) Solve the coercive elliptic problem (2.21) with $g=g^{(0)}$ to determine the new search direction $\tilde{g}^{(0)}$

(4) $\operatorname{Set} r^{(0)}=d^{(0)}=\tilde{g}^{(0)}$.

(5) For $k=1,2, \ldots$,

5.1. Solve the homogeneous wave equations (2.2) $\left(f=g_{D}=g_{S}=g_{N}=0\right.$ ) with the initial values $d^{(k)}=\left(d_{0}^{(k)}, d_{1}^{(k)}\right)$ and (2.20). Compute the gradient $g^{(k)}=J_{1}^{\prime}\left(d^{(k)}\right)$ defined by (2.19).

5.2. Solve the coercive elliptic problem (2.21) with $g=g^{(k)}$ to get $\tilde{g}^{(k)}$.

5.3. $\alpha_{k}=\frac{\left\|c \nabla r_{0}^{(k)}\right\|_{L^{2}(\Omega)}^{2}+\left\|r_{1}^{(k)}\right\|_{L^{2}(\Omega)}^{2}}{\left(c^{2} \nabla \tilde{g}_{0}^{(k)}, \nabla d_{0}^{(k)}\right)_{L^{2}(\Omega)}+\left(\tilde{g}_{1}^{(k)}, d_{1}^{(k)}\right)_{L^{2}(\Omega)}}$

5.4. $v^{(k+1)}=v^{(k)}-\alpha_{k} d^{(k)}$

5.5. $r^{(k+1)}=r^{(k)}-\alpha_{k} \tilde{g}^{(k)}$

5.6. $\beta_{k}=\frac{\left\|c \nabla r_{0}^{(k+1)}\right\|_{L^{2}(\Omega)}^{2}+\left\|r_{1}^{(k+1)}\right\|_{L^{2}(\Omega)}^{2}}{\left\|c \nabla r_{0}^{(k)}\right\|_{L^{2}(\Omega)}^{2}+\left\|r_{1}^{(k)}\right\|_{L^{2}(\Omega)}^{2}}$

5.7. $\quad d^{(k+1)}=r^{(k+1)}+\beta_{k} d^{(k)}$

5.8. Stop when the relative residual lies below the given tolerance tol

$$
r e s^{(k)}=\sqrt{\frac{\left\|c \nabla r_{0}^{(k+1)}\right\|_{L^{2}(\Omega)}^{2}+\left\|r_{1}^{(k+1)}\right\|_{L^{2}(\Omega)}^{2}}{\left\|c \nabla r_{0}^{(0)}\right\|_{L^{2}(\Omega)}^{2}+\left\|r_{1}^{(0)}\right\|_{L^{2}(\Omega)}^{2}}} \leqslant \text { tol. }
$$


Since $\tilde{g}_{0} \in H^{1}(\Omega)$, the updates of $r_{0}^{(k)}, d_{0}^{(k)}$ and $v_{0}^{(k)}$ in Steps 5.4, 5.5 and 5.7 in the CM-CG Algorithm also remain in $H^{1}(\Omega)$. We emphasize that (2.21a) is independent of $\omega$ and leads to a symmetric and positive definite linear system, which can be solved efficiently and in parallel with standard numerical (multigrid, domain decomposition, etc.) methods $[5,7]$.

In $(2.21 \mathrm{a}), \tilde{g}$ corresponds to the Riesz representer of the gradient $J_{1}^{\prime}(v)$ with respect to the inner product associated to the energy (or $H_{D}^{1}$ semi-) norm. In the absence of Dirichlet boundary conditions, $\Gamma_{D}=\varnothing$, we instead use the Riesz representer corresponding to the full $H^{1}$-norm in Steps 3 and 5.2 of the CM-CG Algorithm.

2.2. Sound-Hard Scattering. Let $u$ be the (unique) solution of the Helmholtz equation (2.1) with $\left|\Gamma_{S}\right|>0$, but now $\Gamma_{D}=\varnothing$, as in a typical sound-hard scattering problem where $\Gamma_{N}$ corresponds to the boundary of the obstacle and $\Gamma_{S}$ to the exterior artificial boundary. Since $\left|\Gamma_{S}\right|>0$, we again immediately conclude from Theorem 1 that $\eta, \alpha_{\ell}$ and $\beta_{\ell}$ identically vanish in (2.8). However, in contrast to the situation of sound-soft scattering from Section 2.1, $\lambda$ is no longer necessarily zero. In fact, Theorem 1 and Remark 1 imply that any (global) minimizer $v$ of $J_{1}$ necessarily corresponds to an arbitrary constant shift of $u$, i.e. $v=u+\lambda, \lambda \in \mathbb{R}$. To remove the spurious constant shift and restore uniqueness, we propose three distinct approaches:

(i) determine $\lambda$ by enforcing a compatibility condition,

(ii) replace $J_{1}$ by an alternative functional or

(iii) use an absorbing boundary condition on $\Gamma_{S}$ with a zeroth order term.

(i) Compatibility condition. From Theorem 1 , we know that if $J_{1}\left(v_{0}, v_{1}\right)=0$ there exists a constant $\lambda \in \mathbb{R}$ such that

$$
v(x)=v_{0}(x)+\frac{i}{\omega} v_{1}(x)=u(x)+\lambda .
$$

However, since $\left|\Gamma_{S}\right|>0,(2.1)$ always has a unique solution $u$. Hence, we shall now derive an additional condition from (2.1) to determine $\lambda$.

By integrating (2.1a) and using the boundary conditions (2.1b) and (2.1c), we obtain

$$
\begin{aligned}
0= & -\omega^{2} \int_{\Omega} u(x) d x-\int_{\Omega} \nabla \cdot\left(c^{2}(x) \nabla u(x)\right) d x-\int_{\Omega} f(x) d x \\
= & -\omega^{2} \int_{\Omega} u(x) d x-\int_{\partial \Omega} c^{2}(x) \frac{\partial u(x)}{\partial \nu} d s-\int_{\Omega} f(x) d x \\
= & -\omega^{2} \int_{\Omega} u(x) d x-i \omega c_{0} \int_{\Gamma_{S}} u(x) d s \\
& -\int_{\Omega} f(x) d x-c_{0}^{2} \int_{\Gamma_{S}} g_{S}(x) d s-\int_{\Gamma_{N}} c^{2}(x) g_{N}(x) d s .
\end{aligned}
$$

Replacing $u$ by $v-\lambda$ in (2.22) yields after some algebra the compatibility condition

$$
\lambda=\frac{-\omega^{2} \int_{\Omega} v-i \omega c_{0} \int_{\Gamma_{S}} v-\int_{\Omega} f d x-c_{0}^{2} \int_{\Gamma_{S}} g_{S}-\int_{\Gamma_{N}} c^{2} g_{N}}{\omega^{2}|\Omega|+i \omega c_{0}\left|\Gamma_{S}\right|} .
$$




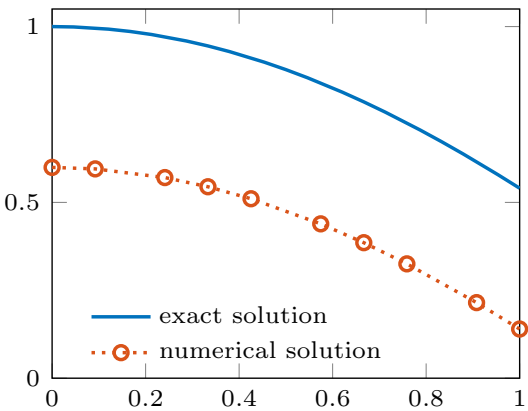

(a) without compatibility condition

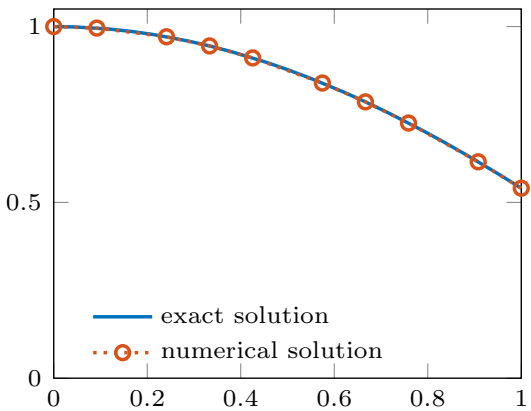

(b) with compatibility condition

Figure 2. One-dimensional Helmholtz equation (2.1) with Neumann condition at $x=0$ and Sommerfeld condition at $x=1$. Exact and numerical solution using the CM-CG method with the cost functional $J_{1}$; left: without compatibility condition, right: with compatibility condition (2.23)-(2.24).

Thus, once we have found a $T$-periodic solution $v=v_{0}+(i / \omega) v_{1}$ of $(2.2)$, we determine the correct (unique) time-harmonic solution $u$ of (2.1) simply by applying the constant shift

$$
u=v-\lambda,
$$

with $\lambda$ given by (2.23).

To illustrate the usefulness of the compatibility condition (2.23)-(2.24), we now consider the following simple one-dimensional example. Let $u(x)=\mathrm{e}^{i x}$ be the exact solution of $(2.1)$ in $\Omega=(0,1)$ with $\Gamma_{N}=\{0\}, \Gamma_{S}=\{1\}, \omega=c=1$, and $f \equiv 0$. In Figure 2, we display the exact and the numerical solution obtained with the CMCG method using the functional $J_{1}$ in (2.4). The CM-CG iteration converges to a minimizer $v$, which contains a spurious constant shift from $u$. By computing $\lambda$ in (2.23) and subtracting it from $v$, we recover the exact solution $u$, as shown in Figure $2 \mathrm{~b}$.

(ii) Alternative functionals. Since the coerciveness of $J_{1}$ in (2.4) "depends in a subtle way on the geometry of $\Omega$ " [11], Bardos and Rauch proposed an alternative cost functional, $J_{\infty}$, which is coercive even in situations with trapping rays. In [8], Bristeau et al. used the following real-valued version of $J_{\infty}$ :

$$
\begin{aligned}
J_{\infty}\left(v_{0}, v_{1}\right)= & \frac{1}{2} \int_{0}^{T} \int_{\Omega}\left|c(x) \nabla\left(y(x, t)-\operatorname{Re}\left\{v(x) \mathrm{e}^{-i \omega t}\right\}\right)\right|^{2} d x d t \\
& +\frac{1}{2} \int_{0}^{T} \int_{\Omega}\left(y_{t}(x, T)-\omega \operatorname{Im}\left\{v(x) \mathrm{e}^{-i \omega t}\right\}\right)^{2} d x d t,
\end{aligned}
$$

where $y$ solves $(2.2)$ and $v=v_{0}+(i / \omega) v_{1}$. In contrast to $J_{1}$, the functional $J_{\infty}$ penalizes the departure from the desired time-harmonic behavior not only after one period at $t=T$, but in fact at all times. In (Section 7.6.2, [8]), the CM-CG method with $J_{1}$ or $J_{\infty}$ was applied to wave scattering from a perfectly conducting circular cavity 
with a crack and the numerical "solutions obtained with the two control approaches were the same".

When the cost functional $J_{1}$ is replaced by $J_{\infty}$ in the CM-CG Algorithm (Section 2.1), the Fréchet derivative of $J_{\infty}$ requires the solution of modified forward and backward wave equations akin to (2.2) and (2.20). However, unlike in the original CM-CG approach with $J_{1}$, the backward wave equation now depends explicitly on the entire solution of the forward wave equation. To avoid storing the entire history of the forward wave equation, which may be prohibitive for large-scale problems, we consider instead the alternative cost functional

$$
\begin{aligned}
J_{m}\left(v_{0}, v_{1}\right)= & \frac{1}{2} \sum_{j=1}^{m} \int_{\Omega}\left|c(x) \nabla\left(y\left(x, t_{j}\right)-\operatorname{Re}\left\{v(x) \mathrm{e}^{-i \omega t_{j}}\right\}\right)\right|^{2} d x \\
& +\frac{1}{2} \sum_{j=1}^{m} \int_{\Omega}\left(y_{t}\left(x, t_{j}\right)-\omega \operatorname{Im}\left\{v(x) \mathrm{e}^{-i \omega t_{j}}\right\}\right)^{2} d x, \quad m \geqslant 1,
\end{aligned}
$$

where $y$ solves $(2.2)$ and $v=v_{0}+(i / \omega) v_{1}$. Here $0<t_{1}<t_{2}<\ldots<t_{m}=T$ are $m$ fixed time instants - we shall only use $m \leqslant 3$. Note that $J_{m}$ coincides with $J_{1}$ for $m=1$ and $t_{1}=T$. In general, the uniqueness of a global minimizer of $J_{m}$ depends on the specific boundary condition in (2.1) and on the choice of $\left\{t_{j}\right\}_{j=1}^{m}$. In particular, for $\Gamma_{D}=\varnothing$ and $\left|\Gamma_{S}\right|>0$, the functional $J_{2}$ in (2.26) with $t_{1}=T / 4$ and $t_{2}=T$ always has a unique global minimizer.

Theorem 2. Let $u \in H^{1}(\Omega)$ be the unique solution of (2.1) with $\Gamma_{D}=\varnothing$ and $\left|\Gamma_{S}\right|>0$. For $\left(v_{0}, v_{1}\right) \in H^{1}(\Omega) \times H^{1}(\Omega)$, the following assertions are equivalent:

(i) $u=v_{0}+(i / \omega) v_{1}$.

(ii) $J_{2}\left(v_{0}, v_{1}\right)=0$ with $J_{2}$ as in (2.26), $t_{1}=T / 4$ and $t_{2}=T$.

Proof. The implication (i) $\Rightarrow$ (ii) obviously holds, since the solution $y$ of (2.2) with initial values $\left(v_{0}, v_{1}\right)=(\operatorname{Re}\{u\}, \omega \operatorname{Im}\{u\})$ is unique and equal to $\operatorname{Re}\left\{u(x) \mathrm{e}^{-i \omega t}\right\}$.

To show that (ii) $\Rightarrow(\mathrm{i})$, let $\left(v_{0}, v_{1}\right) \in H^{1}(\Omega) \times H^{1}(\Omega)$ be a minimizer of $J_{2}$ and $v=v_{0}+(i / \omega) v_{1}$. Then, for the corresponding solution $y$ of $(2.2)$,

$$
\nabla(y(x, T)-y(x, 0))=0, \quad y_{t}(x, T)-y_{t}(x, 0)=0 \quad \text { in } \Omega
$$

and thus both $\nabla y$ and $y_{t}$ are $T$-periodic. From Theorem 1 and Remark 1 , we infer that

$$
y(x, t)=\operatorname{Re}\left\{u(x) \mathrm{e}^{-i \omega t}\right\}+\lambda
$$

for some constant $\lambda \in \mathbb{R}$ and that $v=u+\lambda$. Thus

$$
y(x, t)=\operatorname{Re}\left\{(v(x)-\lambda) \mathrm{e}^{-i \omega t}\right\}+\lambda=\operatorname{Re}\left\{v(x) \mathrm{e}^{-i \omega t}\right\}+\lambda(1-\cos (\omega t)) .
$$

Since $J_{2}\left(v_{0}, v_{1}\right)=0$, we also have

$$
y_{t}\left(x, \frac{T}{4}\right)-\omega \operatorname{Im}\left\{v(x) \mathrm{e}^{-i \omega \frac{T}{4}}\right\}=0, \quad x \in \Omega .
$$

We differentiate $(2.27)$ in time, set $t=T / 4$ and use (2.28) to obtain

$$
0=y_{t}\left(x, \frac{T}{4}\right)-\omega \operatorname{Im}\left\{v(x) \mathrm{e}^{-i \omega \frac{T}{4}}\right\}-\omega \lambda \sin \left(\frac{\omega T}{4}\right)=-\omega \lambda \sin \left(\frac{\pi}{2}\right)=-\omega \lambda .
$$


Since $\omega>0,(2.29)$ implies that $\lambda=0$ and hence that $u=v$, which concludes the proof.

To apply the CM-CG method with the alternative functional $J_{m}$, we need to compute its Fréchet derivative. Let $v=\left(v_{0}, v_{1}\right) \in H^{1}(\Omega) \times H^{1}(\Omega), \delta v=\left(\delta v_{0}, \delta v_{1}\right)$ be an arbitrary perturbation and define

$$
\begin{aligned}
\widetilde{v}(x, t)=\operatorname{Re}\left\{\left(v_{0}(x)+\frac{i}{\omega} v_{1}(x)\right) \mathrm{e}^{-i \omega t}\right\}, & x \in \Omega, t>0, \\
\widetilde{\delta v}(x, t)=\operatorname{Re}\left\{\left(\delta v_{0}(x)+\frac{i}{\omega} \delta v_{1}(x)\right) \mathrm{e}^{-i \omega t}\right\}, & x \in \Omega, t>0 .
\end{aligned}
$$

Then, the Fréchet derivative of $J_{m}$ is

$$
\begin{aligned}
\left\langle J_{m}^{\prime}(v), \delta v\right\rangle= & -\sum_{\ell=1}^{m} \int_{\Omega} c^{2}(x) \nabla\left(y\left(x, t_{\ell}\right)-\widetilde{v}\left(x, t_{\ell}\right)\right) \nabla \widetilde{\delta v}\left(x, t_{\ell}\right) d x \\
& -\sum_{\ell=1}^{m} \int_{\Omega}\left(y_{t}\left(x, t_{\ell}\right)-\widetilde{v}_{t}\left(x, t_{\ell}\right)\right) \widetilde{\delta v_{t}}\left(x, t_{\ell}\right) d x \\
& +\int_{\Omega}\left(p(x, 0) \delta v_{1}(x)-p_{t}(x, 0) \delta v_{0}(x)\right) d x+c_{0} \int_{\Gamma_{S}} p(x, 0) \delta v_{0}(x) d s .
\end{aligned}
$$

Here $p=\sum_{\ell=1}^{m} p^{\ell}$ and each $p^{\ell}$ solves the adjoint (backward) wave equation (2.20) with the initial conditions $p^{\ell}\left(x, t_{\ell}\right)=p_{0}^{\ell}(x)$ and $p_{t}^{\ell}\left(x, t_{\ell}\right)=p_{1}^{\ell}(x), 1 \leqslant \ell \leqslant m$, defined by

$$
\begin{aligned}
p_{0}^{\ell}(x)= & y_{t}\left(x, t_{\ell}\right)-\tilde{v}_{t}\left(x, t_{\ell}\right), \quad x \in \Omega, \\
\int_{\Omega} p_{1}^{\ell}(x) w(x) d x= & c_{0} \int_{\Gamma_{S}} p_{0}^{\ell}(x) w(x) d s \\
& -\int_{\Omega} c^{2}(x) \nabla\left(y\left(x, t_{\ell}\right)-\tilde{v}\left(x, t_{\ell}\right)\right) \nabla w(x) d x, \quad \forall w \in H^{1}(\Omega) .
\end{aligned}
$$

The CM-CG method with the cost functional $J_{m}$ in (2.30) requires the solution of a single forward wave equation until time $T$ and $m$ independent backward wave equations over $\left[0, t_{\ell}\right], \ell=1, \ldots, m$. To ensure that both $v_{0}^{(k)}$ and $v_{1}^{(k)}$ remain in $H^{1}(\Omega)$ during the CM-CG iteration, we now determine in Step 3 and 5.2 an explicit Riesz representer $\tilde{g}^{(k)}=\left(\tilde{g}_{0}^{(k)}, \tilde{g}_{1}^{(k)}\right) \in H^{1}(\Omega) \times H^{1}(\Omega)$ of $g^{(k)}=J_{m}^{\prime}\left(v^{(k)}\right)$ by solving an elliptic coercive problem similar to $(2.21)$ for both $\tilde{g}_{0}^{(k)}$ and $\tilde{g}_{1}^{(k)}$. For $m=2, t_{1}=T / 4$ and $t_{2}=T$, for instance, the CM-CG Algorithm based on the functional $J_{2}$ always computes the correct minimizer at a modest $12.5 \%$ increase in the computational cost over using $J_{1}$.

(iii) Absorbing boundary condition with zeroth order term. From Remark 1, we know that the eigenvalue problems (2.10) with $\Gamma_{D}=\varnothing,\left|\Gamma_{S}\right|>0$ and the Sommerfeld condition (2.10b) only have the trivial solution for $\ell \neq 0$. However, we may also replace the simple Sommerfeld-like impedance condition (2.1b) by the (more accurate) first-order Bayliss-Gunzburger-Turkel (BGT-1) condition [24,25] in $d=2,3$ 
dimensions, for instance:

$$
\frac{\partial u(x)}{\partial \nu}-\frac{i \omega}{c_{0}} u(x)+\frac{1}{(4-d) r} u(x)=g_{S}(x), \quad x \in \Gamma_{S} .
$$

The corresponding time-harmonic wave field $y$ then solves (2.2) with (2.2b) replaced by

$$
\frac{\partial y(x, t)}{\partial \nu}+\frac{1}{c_{0}} \frac{\partial y(x, t)}{\partial t}+\frac{1}{(4-d) r} y(x, t)=\operatorname{Re}\left\{g_{S}(x) \mathrm{e}^{-i \omega t}\right\}, \quad x \in \Gamma_{S}, t>0,
$$

while Theorem 1 and Remark 1 still hold with (2.10b) replaced by

$$
\frac{\partial \gamma_{\ell}(x)}{\partial \nu}-\frac{i \omega \ell}{c_{0}} \gamma_{\ell}(x)+\frac{1}{(4-d) r} \gamma_{\ell}(x)=0, \quad x \in \Gamma_{S},
$$

and similarly for $\alpha_{\ell}, \beta_{\ell}$ in (2.6c) and (2.7c). Because of the strictly positive (or negative) definite imaginary part of the zeroth order coefficient in $(2.31 \mathrm{~b})$, the eigenvalue problems (2.10) still only have the trivial solution for $\ell \neq 0$ [25]. Moreover even for $\ell=0$, the nonzero coefficient of the zeroth order remaining term in $(2.32)$ now implies that $\gamma_{0}$ is not only constant but in fact zero everywhere in $\Omega$. In this case, the $T$-periodicity of $y$ is sufficient to guarantee convergence to the (unique) solution of the Helmholtz equation (2.1). Clearly, other absorbing boundary conditions [25] may also permit the use of $J_{1}$ without any modification.

\section{Controllability Method in physically Bounded domains}

In Sections 2.1 and 2.2, we considered various scattering problems where we impose an impedance boundary condition $(2.1 b)$ on part of the boundary $\Gamma_{S}$. Here, we consider the Helmholtz equation (2.1) with $\Gamma_{S}=\varnothing$, i.e. with pure Dirichlet or Neumann boundary condition. Clearly, to guarantee the uniqueness of the solution $u$ of (2.1), we now always assume that $\omega^{2}$ is not an eigenvalue of the principal elliptic part. Still, the CM-CG method with the cost functional $J_{1}$ from (2.4) in general will not yield the correct solution $u$, not even shifted by an arbitrary constant. Indeed, let $\left(v_{0}, v_{1}\right) \in H^{1}(\Omega) \times L^{2}(\Omega)$ be a minimizer of $J_{1}$. According to Theorem $1, v=$ $v_{0}+(i / \omega) v_{1}$ then satisfies (2.8) with $\alpha_{\ell}, \beta_{\ell}$ solutions of (2.6) and (2.7). While for $\left|\Gamma_{S}\right|>0$ all eigenfunctions $\alpha_{\ell}, \beta_{\ell}, \ell \geqslant 1$, necessarily vanish (Remark 1 ), this is no longer the case when $\Gamma_{S}=\varnothing$, as $(\omega \ell)^{2}$ may be an eigenvalue of the principal part for some $\ell \geqslant 1$, even when $\omega^{2}$ is not.

To illustrate this added ambiguity, we now consider the following simple onedimensional example. Let $u(x)=4 x(1-x)$ be the solution of $(2.1)$ in $\Omega=(0,1)$ with $c=1, \omega=\pi / 4$ and Neumann boundary conditions $u^{\prime}(0)=4$ and $u^{\prime}(1)=-4$. Since $\omega^{2}$ does not lie in the spectrum $\Sigma=\left\{(\ell \pi)^{2}: \ell \in \mathbb{Z}\right\}$ of $(2.10),(2.1)$ is well-posed. However, $(\omega \ell)^{2} \in \Sigma$ for any $\ell \in 4 \mathbb{Z}$ with corresponding eigenfunction $\alpha_{\ell}(x)=\cos (\omega \ell x)$. Thus

$$
J_{1}\left(v_{0}+\alpha_{\ell}, v_{1}\right)=J_{1}\left(v_{0}, v_{1}\right), \quad\left(v_{0}, v_{1}\right) \in H^{1}(\Omega) \times L^{2}(\Omega), \ell \in 4 \mathbb{Z},
$$

and minimizers of $J_{1}$ are neither unique nor do they simply differ by a constant. As shown in Figure $3 \mathrm{a}$ and $3 \mathrm{~b}$, the CM-CG method using $J_{1}$, or even $J_{2}$, does not yield 


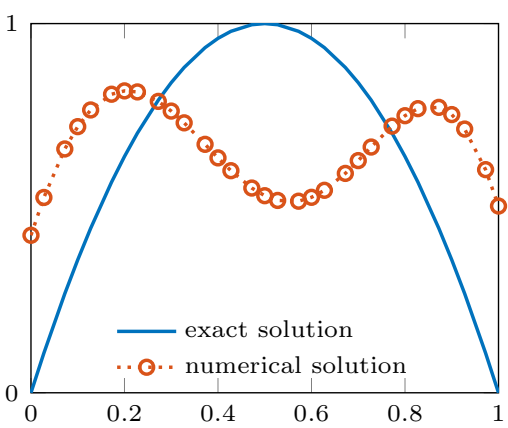

(a) $J_{1}($ rel. error $=27 \%)$

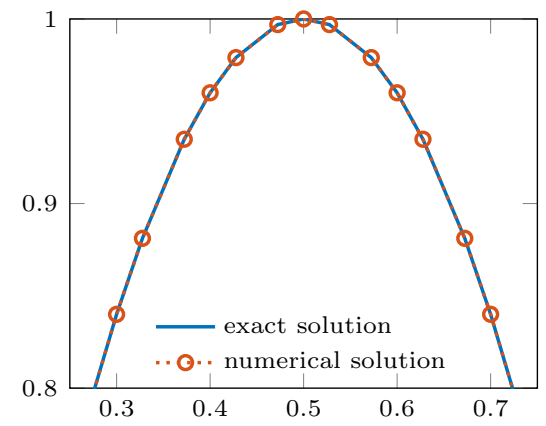

(c) $J_{3}$ (rel. error $\left.=4.4 \cdot 10^{-7 \%}\right)$

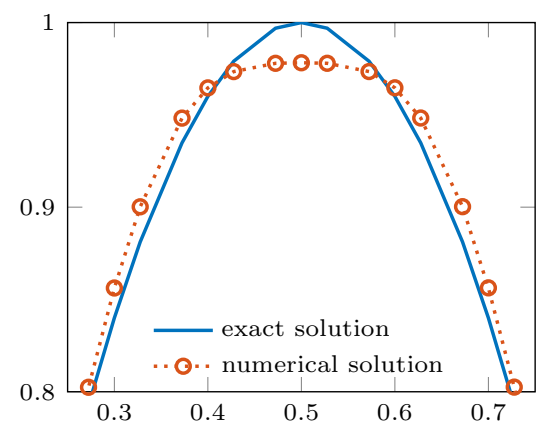

(b) $J_{2}$ (rel. error $=2.1 \%$ )

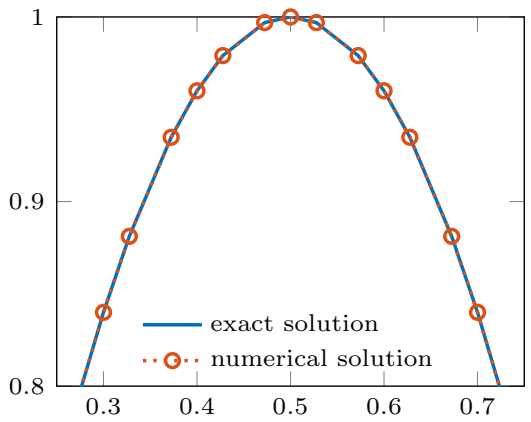

(d) $J_{\infty}\left(\right.$ rel. error $\left.=3.4 \cdot 10^{-7 \%}\right)$

Figure 3. Helmholtz equation in a bounded domain: numerical solutions of $(2.1)$ in $\Omega=(0,1)$ with Neumann conditions at $x=0,1$ using the CM-CG method either with $J_{1}$ (top left), $J_{2}$ (top right), $J_{3}$ (bottom left) or $J_{\infty}$ (bottom right)

the correct minimizer $u$. However, the cost functional $J_{\infty}$ in (2.25) remains strictly convex; hence, the CM-CG method based on $J_{\infty}$ converges to the correct minimizer $u$, as shown in Figure 3d. Similarly, the cost functional $J_{3}$ in $(2.26)$ with $t_{1}=T / 4$, $t_{2}=T / 2$ and $t_{3}=T$ always yields $u$, as illustrated in Figure $3 \mathrm{c}$ and shown in the following theorem.

Theorem 3. Let $u \in H^{1}(\Omega)$ be the unique solution of the Helmholtz equation (2.1) and $\left(v_{0}, v_{1}\right) \in H^{1}(\Omega) \times H^{1}(\Omega)$. The following assertions are equivalent:

(i) $u=v_{0}+(i / \omega) v_{1}$

(ii) $J_{3}\left(v_{0}, v_{1}\right)=0$ with $t_{1}=T / 4, t_{2}=T / 2, t_{3}=T$, and $m=3$ in (2.26).

Proof. As in the proof of Theorem 2, the implication (i) $\Rightarrow$ (ii) immediately holds.

Now, let $\left(v_{0}, v_{1}\right) \in H^{1}(\Omega) \times H^{1}(\Omega)$ be a minimizer of $J_{3}$ and $y$ be the solution of (2.2). Then, $\nabla(y(x, T)-y(x, 0))$ and $y_{t}(x, T)-y_{t}(x, 0)$ vanish identically in $\Omega$. Next, 
we define the "misfit function"

$$
r(x, t)=y(x, t)-\operatorname{Re}\left\{v(x) \mathrm{e}^{-i \omega t}\right\}
$$

and use (2.5) and (2.8) from Theorem 1 to replace $v$ and $y$ in (3.1). This yields

$$
\begin{aligned}
(r(\cdot, t), \varphi)= & \left(y(\cdot, t)-\operatorname{Re}\left\{u \mathrm{e}^{-i \omega t}\right\}, \varphi\right)-\left(\operatorname{Re}\left\{v \mathrm{e}^{-i \omega t}\right\}-\operatorname{Re}\left\{u \mathrm{e}^{-i \omega t}\right\}, \varphi\right) \\
= & \left(\lambda(1-\cos (\omega t))+\eta\left(t-\omega^{-1} \sin (\omega t)\right), \varphi\right) \\
& +\sum_{\ell=1}^{\infty}\left\{\left(\alpha_{\ell}(\cos (\omega \ell t)-\cos (\omega t))+\beta_{\ell}(\sin (\omega \ell t)-\ell \sin (\omega t), \varphi)\right\} .\right.
\end{aligned}
$$

Differentiating once with respect to time, we obtain

$$
\begin{aligned}
\left(r_{t}(\cdot, t), \varphi\right)= & (\omega \lambda \sin (\omega t)+\eta(1-\cos (\omega t)), \varphi) \\
& +\omega \sum_{\ell=1}^{\infty}\left\{\left(-\alpha_{\ell}(\ell \sin (\omega \ell t)-\sin (\omega t))+\ell \beta_{\ell}(\cos (\omega \ell t)-\cos (\omega t)), \varphi\right)\right\} .
\end{aligned}
$$

Since $\nabla r\left(\cdot, t_{j}\right)=0$ and $r_{t}\left(\cdot, t_{j}\right)=0$ for $t_{j} \in\{T / 4, T / 2\}, r\left(\cdot, t_{j}\right) \equiv \mu_{j}$ is constant a.e. in $\Omega, j=1,2$, while for $t=t_{2}=T / 2=\pi / \omega,(3.2)$ and (3.3) reduce to

$$
\begin{aligned}
\left(\mu_{2}, \varphi\right) & =\left(2 \lambda+\eta \frac{T}{2}, \varphi\right)+\sum_{\ell=1}^{\infty}\left((-1)^{\ell}+1\right)\left(\alpha_{\ell}, \varphi\right), \\
0 & =(2 \eta, \varphi)+\omega \sum_{\ell=1}^{\infty} \ell\left((-1)^{\ell}+1\right)\left(\beta_{\ell}, \varphi\right) .
\end{aligned}
$$

Thus,

$$
\left(2 \lambda+\eta \frac{T}{2}-\mu_{2}, \varphi\right)+2 \sum_{\substack{\ell=2 \\ \ell \text { even }}}^{\infty}\left(\alpha_{\ell}, \varphi\right)=(2 \eta, \varphi)+2 \omega \sum_{\substack{\ell=2 \\ \ell \text { even }}}^{\infty} \ell\left(\beta_{\ell}, \varphi\right)=0
$$

for any test function $\varphi \in H_{D}^{1}(\Omega)$.

If $\left|\Gamma_{D}\right|>0, \mu_{1}=\mu_{2}=0$ and $\lambda=\eta=0$ by Theorem 1 . Otherwise if $\Gamma_{D}=\varnothing$, $2 \lambda+\eta T / 2-\mu_{2}$ and $2 \eta$ both solve (2.10) with $\ell=0$ and hence are eigenfunctions with zero eigenvalue unless they vanish identically. Similarly, $\alpha_{\ell}$ and $\beta_{\ell}$ are either eigenfunctions of (2.6) and (2.7) for the eigenvalue $(\omega \ell)^{2}$ or identically zero. By orthogonality, $\eta=0$ and the eigenfunctions $\alpha_{\ell}=0$ and $\beta_{\ell}=0$ for any even $\ell \geqslant 2$.

Now, we set $t=t_{1}=T / 4=\pi /(2 \omega)$ in (3.2) and (3.3) to obtain

$$
\begin{aligned}
\left(\mu_{1}, \varphi\right) & =(\lambda, \varphi)-\sum_{\substack{\ell=1 \\
\ell \text { odd }}}^{\infty}\left((-1)^{\ell}+\ell\right)\left(\beta_{\ell}, \varphi\right)=(\lambda, \varphi)-\sum_{\substack{\ell=3 \\
\ell \text { odd }}}^{\infty}(\ell-1)\left(\beta_{\ell}, \varphi\right), \\
0 & =(\omega \lambda, \varphi)+\omega \sum_{\substack{\ell=1 \\
\ell \text { odd }}}^{\infty}\left(\ell(-1)^{\ell}+1\right)\left(\alpha_{\ell}, \varphi\right)=(\omega \lambda, \varphi)-\omega \sum_{\substack{\ell=3 \\
\ell \text { odd }}}^{\infty}(\ell-1)\left(\alpha_{\ell}, \varphi\right) .
\end{aligned}
$$

If $\alpha_{\ell}$ and $\beta_{\ell}$ do not identically vanish, they are eigenfunctions of (2.6) and (2.7), respectively. Since $\ell-1>0$ for any odd number $\ell \geqslant 3$, the orthogonality of the eigenfunctions implies $\alpha_{\ell}=0$ and $\beta_{\ell}=0$ for any odd $\ell \geqslant 3$ and $\lambda=0$. Hence, $\alpha_{\ell}=\beta_{\ell}=0$ for $\ell \neq 1$ and $\lambda=\eta=0$. Since (2.10) with $\ell=1$ corresponds to (2.1), 


\begin{tabular}{c|ccccc} 
& $J_{1}$ & $J_{1}^{C C}$ & $J_{2}$ & $J_{3}$ & $J_{\infty}$ \\
\hline number of periods [0,T] & 2 & 2 & 2.25 & 2.75 & 2 \\
& & & & & \\
\hline relative computational time & 1 & 1 & 1.125 & 1.375 & 1 \\
& & & & & \\
\hline relative memory requirement & 1 & 1 & 2 & 3 & $n_{T}$ \\
\hline sound-soft scattering & $\checkmark$ & $\checkmark$ & $\checkmark$ & $\checkmark$ & $\checkmark$ \\
sound-hard scattering & $\times$ & $\checkmark$ & $\checkmark$ & $\checkmark$ & $\checkmark$ \\
physically bounded domain & $\times$ & $\times$ & $\times$ & $\checkmark$ & $\checkmark$
\end{tabular}

Table 1. Computational cost and storage of the CM-CG method using the alternative functionals $J_{m}$ or $J_{\infty}$ relative to that using the original functional $J_{1}$. Crosses indicate that the CM-CG method generally does not yield the correct time-harmonic solution. The top line indicates the number of periods $[0, T]$ for which the forward or backward wave equation (2.2) must be solved.

which has a unique solution by assumption, $\alpha_{1}$ and $\beta_{1}$ are also identically zero, which concludes the proof.

In Table 1, we estimate the computational effort and storage of the CM-CG method using different penalty functionals relative to that using the original functional $J_{1}$. Here $n_{T}=T / \Delta t$ denotes the number of time steps in the solution of the forward wave equation over one period $[0, T]$. The functional $J_{m}$ is slightly more expensive for $m=2,3$, because each time instant $t_{j}, 1 \leqslant j \leqslant m$, needs the solution of an adjoint wave equation, but only over $\left[0, t_{j}\right]$. Therefore, the functional $J_{2}$ with $t_{1}=T / 4$ and $t_{2}=T$ requires $n_{T}$ time-steps to obtain the forward solution (2.2) at $t=T$ and $\left(n_{T}+n_{T} / 4\right)$ time-steps for the two adjoint wave equations $(2.20)$, which yields a total of $2.25 \times n_{T}$ time-steps. Similarly, the functional $J_{3}$ with $t_{1}=T / 4, t_{2}=T / 2$ and $t_{3}=T$ requires $n_{T}$ time-steps for the forward solution and $\left(n_{T} / 4+n_{T} / 2+n_{T}\right)$ time-steps for the adjoint solutions, which leads to $2.75 \times n_{T}$ time-steps in total.

In contrast, the functional $J_{\infty}$ only needs the solution of a single forward and a single backward wave equation over $[0, T]$, which yields the same computational effort as the functional $J_{1}$. However, as the adjoint wave equation then involves the entire history of the forward solution, it becomes necessary to store the forward solution at all time steps $t_{j}=j \Delta t, 1 \leqslant j \leqslant n_{T}$.

\section{Numerical Results}

Here we present a series of numerical experiments to illustrate the usefulness of controllability methods (CM-CG) for the solution of the Helmholtz equation (2.1) in various typical configurations. First, we consider a plane wave exact solution and verify that the CM-CG method not only achieves the expected rates of convergence, but also significantly accelerates the convergence to the long-time asymptotic time-harmonic limit. Second, we apply the CM-CG method with the original energy functional $J_{1}$ from $(2.4)$ to a typical sound-soft scattering problem, as in $[1,8]$. To 


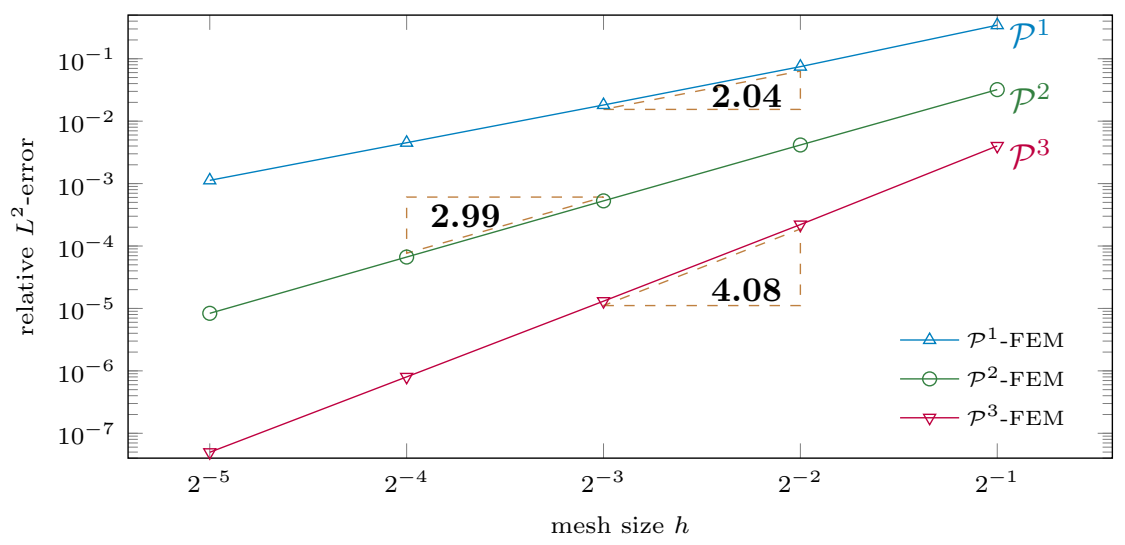

Figure 4. Plane wave solution: the relative error of the numerical solution in the unit square using the CM-CG method for varying mesh size $h$

overcome the bottleneck in the time integration of (2.2) due to local mesh refinement, we replace standard time marching by Runge-Kutta based explicit local time-stepping methods [16]. Third, we apply the CM-CG method to a sound-hard scattering problem, where we restore uniqueness by imposing the compatibility condition $(2.22)$. We also apply the CM-CG method to scattering from an inhomogeneous inclusion with a first-order absorbing boundary condition [24] to demonstrate that uniqueness is then inherently guaranteed (see Section 2.2). Finally, we show how the CM-CG method can also be efficiently applied in a physically bounded domain by using the functional $J_{3}$ in (2.26). For spatial discretization, we always use standard piecewise polynomial triangular FE with high-order mass-lumping [26].

4.1. Plane Wave Solution. First, we verify that the controllability method with the functional $J_{1}$ from (2.4) indeed achieves the same rate of convergence as the direct solution of the Helmholtz equation. Here, we consider a plane wave solution

$$
u(x, y)=\exp (i k(x \cos (\phi)+y \sin (\phi)),
$$

with the angle of incidence $\phi=135^{\circ}$ and wave number $k=\omega=2 \pi(c \equiv 1)$. Hence we consider $(2.1)$ in the unit square $\Omega=(0,1) \times(0,1)$ and set the boundary conditions accordingly with $\Gamma_{D}=[0,1] \times\{0,1\}$ and $\Gamma_{S}=\{0,1\} \times(0,1)$. We use $\mathcal{P}^{r}$-FE for the spatial discretization with $r=1, \ldots, 3$ and the classical fourth-order RungeKutta (RK4) method for the time integration of (2.2). The tolerance in the CM-CG Algorithm (Section 2.1) is set to tol $=10^{-11}$.

In Figure 4, we observe that the relative error of the numerical solution, obtained with the CM-CG method for a sequence of meshes with mesh size $h=2^{-\ell}$, $\ell=1, \ldots, 5$, converges with the (expected) optimal rate as $h^{r+1}$ with respect to the $L^{2}$-norm. Clearly, as the mesh size decreases we also reduce the time-step $\Delta t$ for numerical stability. 


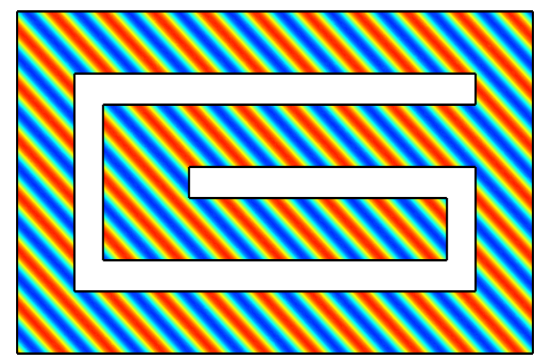

(a) exact solution

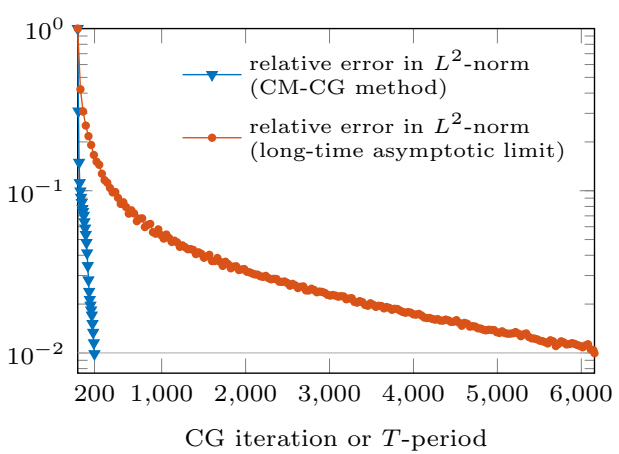

(b) relative error

Figure 5. Plane wave solution: the exact solution (a) of the sound-soft scattering problem (2.1) on a rectangular domain with a spiral-shaped cavity; the relative error of the numerical solution obtained either by the CM-CG method at each CG iteration or by simple time integration of (2.2) after the same number of time periods (b)

Next, we show how the CM-CG method greatly accelerates the convergence of the time-harmonically forced solution $y$ of (2.2) to its long-time asymptotic limit $[11,12]$

$$
y(x, t) \sim \operatorname{Re}\{u(x) \exp (-i \omega t)\} \quad \text { as } t \rightarrow+\infty .
$$

To do so, we consider a plane wave (4.1) with angle $\phi=45^{\circ}$ and wave number $k=\omega=32 \pi(c \equiv 1)$ in a rectangular domain with a spiral-shaped cavity - see Figure 5a. We set the boundary conditions accordingly, where $\Gamma_{S}$ is the exterior boundary and $\Gamma_{D}$ the boundary of the spiral-shaped obstacle. Here we use a $\mathcal{P}^{3}$-FE discretization in space and the RK4 method for solving (2.2).

In Figure 5, we compare the relative error in the numerical solution obtained either by the CM-CG approach at the $j$-th CG iteration or by simple time integration of (2.2) at times $t_{j}=j T$, where $T=2 \pi / \omega$ is the time period. We observe that the CM-CG method achieves less than 1\% error after $j=200 \mathrm{CG}$ iterations, whereas the harmonically forced long-time solution of the wave equation requires over 6000 periods to reach the same accuracy.

4.2. Sound-Soft Scattering. Next, we apply the CM-CG method to a typical sound-soft scattering problem from the open wedge shown in Figure 6. Hence we consider (2.1) with $\omega=36 \pi, c \equiv 1, \Gamma_{D}$ the boundary of the wedge $\left(g_{D}=0\right)$ and $\Gamma_{N}=\varnothing$. The unbounded exterior is truncated by a square artificial boundary $\Gamma_{S}$, where we impose the absorbing boundary condition $(2.1 \mathrm{~b})$ with $g_{S}=0$. The initial conditions are set to zero, while the incident wave originates from three point sources located at $(0.75,0.4),(0.5,0.65)$ and $(0.75,0.65)$ in the top right corner of $\Omega$.

For spatial discretization, we use $\mathcal{P}^{3}$-FE with mass-lumping [26] and the mesh shown in Figure 6 with 79917 nodes and 12116 triangles. To overcome the bottleneck from the CFL-restriction on the time step $\Delta t$ due to local mesh refinement near the 


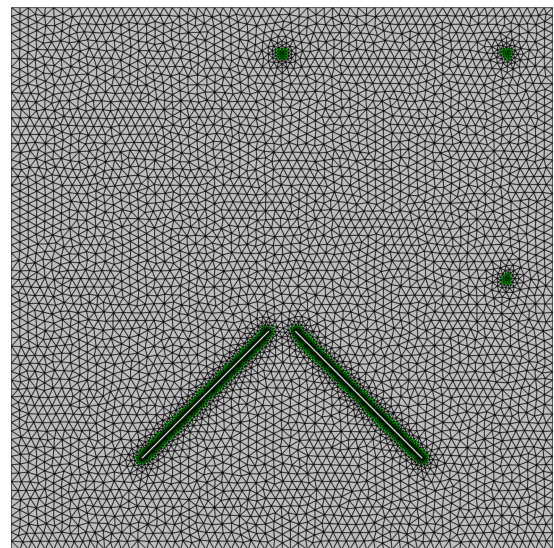

(a) full mesh

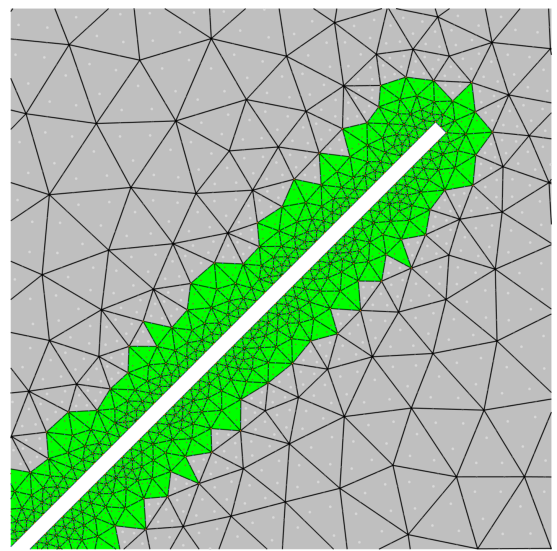

(b) zoom on the refined part of the mesh

Figure 6. Sound-soft scattering: computational mesh with local refinement near the open wedge obstacle and the three point sources

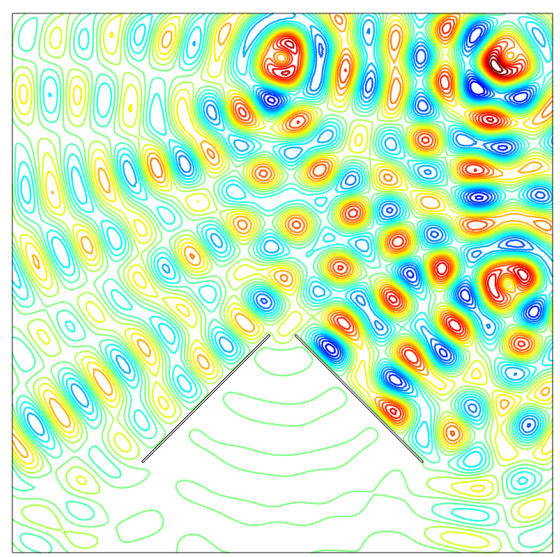

Figure 7. Sound-soft scattering: contour lines of the numerical solution with the CM-CG method combined with explicit local time-stepping.

wedge, we opt for local time-stepping (LTS) methods based on the classical fourthorder RK method [16]. Hence, we split the mesh into "fine" and "coarse" elements see Figure 6 - and use small local time steps of size $\Delta \tau=\Delta t / q$ but only in the "fine" part. Here, $q$ denotes the refinement ratio between the smallest mesh size $h_{\text {min }}^{\text {coarse in }}$

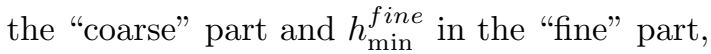

$$
q \approx \frac{h_{\mathrm{min}}^{\text {coarse }}}{h_{\mathrm{min}}^{\text {fine }}} .
$$



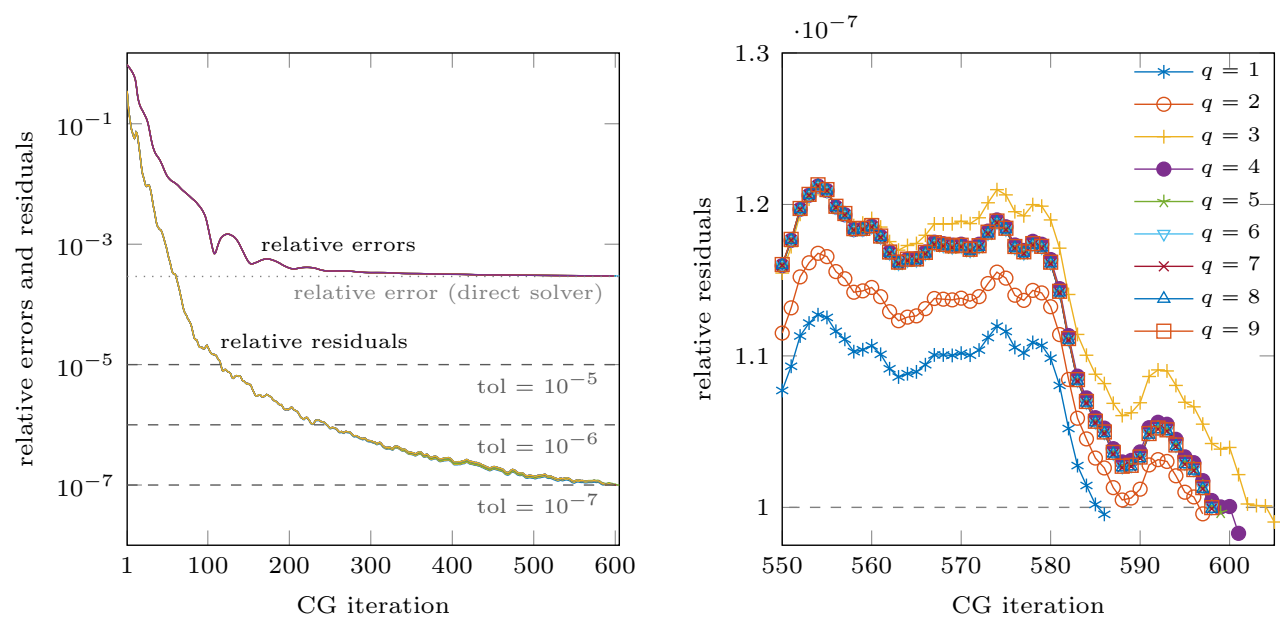

Figure 8. Sound-soft scattering: relative residual and relative error at each CG iteration using the CM-CG method combined with a local time-stepping method with a time-step $\Delta \tau=\Delta t / q$ with $q=1, \ldots, 9$ (left); zoom on the relative residuals at each $\mathrm{CG}$ iteration for varying $q$ (right). In the left frame the curves for different $q$ essentially coincide at this scale.

Figure 7 displays the contour lines of the numerical solution of (2.1) obtained by the CM-CG Algorithm (Section 2.1) with the functional $J_{1}$ from (2.4) and a tolerance tol $=10^{-7}$. The numerical solution obtained with the CM-CG method differs by only $0.15 \%$ from that obtained through direct solution of the Helmholtz equation (2.1). Both solutions differ from a reference solution on a finer mesh by less than $3 \%$.

Now, we combine the CM-CG method with local time-stepping using different mesh refinement ratios $q$. For a fixed mesh, shown in Figure 6, we vary the partition into "fine" and "coarse" elements such that $q=1, \ldots, 9$ in (4.3). Regardless of the tolerance $t o l=10^{-5}, 10^{-6}, 10^{-7}$ in the CM-CG Algorithm, we observe in Figure 8 that the relative residuals and the numbers of iterations remain identical independently of $q$. In summary, the CM-CG method yields a comparable accuracy to the direct solution of the Helmholtz equation while the convergence of the CM-CG method remains unaffected by the local time-stepping strategy.

4.3. Sound-Hard Scattering. Here we apply the CM-CG method to the tpyical situation of an incident plane wave scattered from a sound-hard cavity, as shown in Figure 9. Hence we consider (2.1) with $\Gamma_{D}=\varnothing, \Gamma_{N}$ the boundary of the obstacle $\left(g_{N}=0\right)$ and $\Gamma_{S}$ the exterior square artificial boundary.

Since $\Gamma_{D}=\varnothing$, the original cost functional $J_{1}$ in (2.4) does not have a unique minimizer. To remove the spurious constant shift and thus obtain the correct (unique) solution, shown in Figure 9, we post-process the solution obtained with $J_{1}$ by applying the compatibility condition (2.22) derived in Section 2.2 (i). Here, the angle of the incident wave (4.1) is $\phi=315^{\circ}$ with $c=1$ and $\omega=8 \pi$ whereas the mesh in $\Omega=$ 


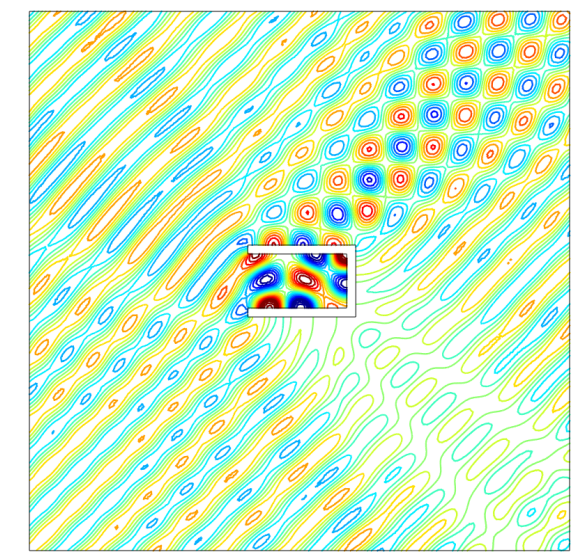

Figure 9. Sound-hard scattering: contour lines of the total wave field obtained with the CM-CG method and the compatibility condition (2.22).

$(0,3) \times(0,3)$ consists of $8146 \mathcal{P}^{3}$-FE with 53456 nodes. For the sake of comparison, we compute a reference solution by solving the Helmholtz equation directly on a finer mesh. The CM-CG method yields a relative error of $0.27 \%$ with a tolerance tol $=10^{-6}$, comparable to that in the direct solution of $(2.1)$ on the same mesh with a relative error of $0.29 \%$.

4.4. Scattering from Inhomogeneous Inclusion. Next, we consider scattering from a penetrable inhomogeneous inclusion with a circular artificial boundary $\Gamma_{S}$; hence, we consider (2.1) with $\Gamma_{D}=\Gamma_{N}=\varnothing$. Instead of applying the compatibility condition (2.22), we enforce uniqueness by replacing the Sommerfeld-like condition $(2.1 \mathrm{~b})$ and $(2.2 \mathrm{~b})$ on $\Gamma_{S}$ by the more accurate first-order Bayliss-Gunzburger-Turkel (BGT-1) absorbing condition (2.31a) and (2.31b), respectively - see Section 2.2 (iii).

Again, we use $\mathcal{P}^{3}$-FEM with 667225 nodes and 102504 elements and apply the RK4 method for the time integration of (2.2). The frequency $\omega=32 \pi$ and the squared propagation speed $c^{2}(x)=c_{0}^{2}+8 \cdot H(x)$, where $H(x)$ is the indicator function of the kite-shaped inclusion and $c_{0}=1$ - see Figure 10 (left). In the right frame of Figure 10, we display the scattered field for an incident plane wave (4.1) with $\phi=180^{\circ}$ obtained by the CM-CG method using the original functional $J_{1}$ from (2.4). Both the numerical solution obtained with the CM-CG method and that obtained by solving (2.1) directly yield a $2.1 \%$ relative error with respect to a reference solution computed on a finer mesh. Thus, the BGT-1 condition, like any other absorbing boundary condition with a positive (or negative) definite zeroth order term, permits the use of the CM-CG approach with the original cost functional $J_{1}$ even when $\Gamma_{D}=\Gamma_{N}=\varnothing$.

4.5. Closed Wave Guide. Finally, we consider the Helmholtz equation (2.1) in a physically bounded domain $\Omega=(-5,1) \times(-0.5,0.5)$ without any impedance boundary condition, $\Gamma_{S}=\varnothing$. At the right vertical entry boundary $x=1$, the wave field satisfies the Dirichlet boundary condition (2.1d) with $g \equiv 1$; elsewhere, it satisfies 


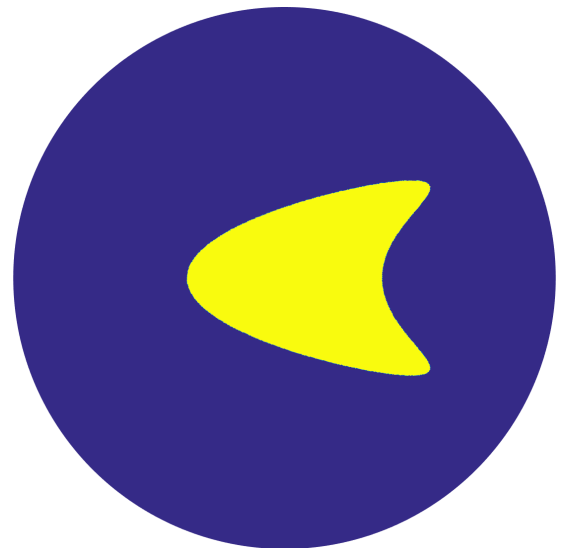

(a) computational domain with inhomogeneous inclusion

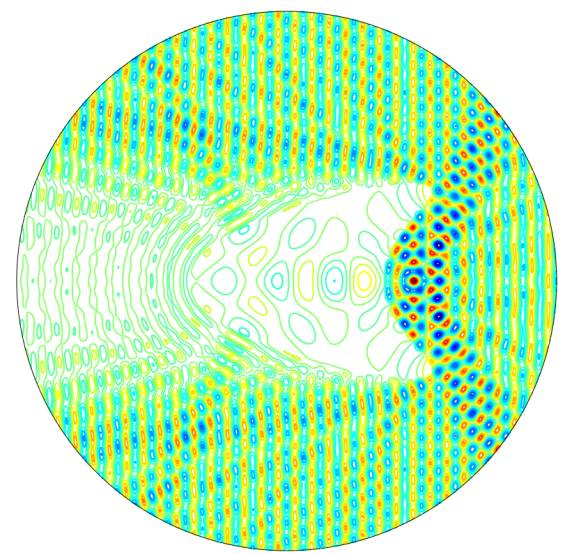

(b) scattered field

Figure 10. Scattering from a penetrable inhomogeneous inclusion: the propagation speed $c$ is constant inside and outside of the kite-shaped inclusion (left); contour lines of the numerical solution obtained with the CM-CG method using the BGT-1 absorbing condition (2.31) and the functional $J_{1}$ (right).

a homogeneous Neumann boundary condition. Here, the frequency $\omega=25$ and the squared propagation speed is $c^{2}(x)=c_{0}^{2}+H(x)$, where $H$ is the indicator function of the ellipse-shaped inclusion shown in Figure 11 (top) and $c_{0}=1$.

Again, we use $\mathcal{P}^{3}$-FEM with 4622 triangles and 30518 nodes for the spatial discretization and the RK4 method for the time integration of (2.2). Here, the solution obtained using the CM-CG method and the cost functional $J_{3}$ in $(2.26)$ with a relative error of $5.7 \%$ is slightly more accurate than that obtained by the direct solution of the linear system with a relative error of $6.3 \%$, probably due to the high condition number of the discrete Helmholtz problem.

\section{Concluding Remarks}

Starting from the original controllability (CM-CG) method for sound-soft scattering problems [4], we have shown how to extend the controllability approach to boundary-value problems governed by the Helmholtz equation with arbitrary combinations of Dirichlet, Neumann or impedance (or Sommerfeld-like) boundary conditions, while remaining numerically efficient.

Akin to a shooting method, the CM-CG method determines the time-harmonic solution of the corresponding wave equation in the time domain by iteratively reducing the departure from periodicity. Each conjugate gradient (CG) iteration then requires the numerical solution of a forward and a backward (or adjoint) wave equation over one period $[0, T], T=2 \pi / \omega$, together with the solution of a coercive elliptic problem independent of the frequency $\omega>0$. Both the wave equation and the symmetric and positive definite linear system required at each CG iteration can be efficiently 


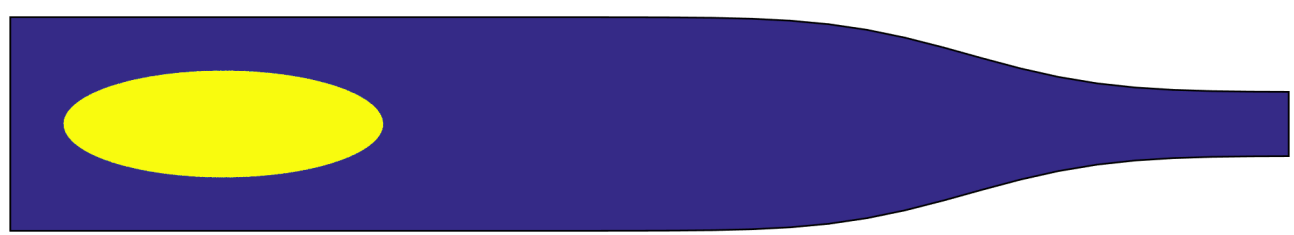

(a) physically bounded domain with an inhomogeneous medium

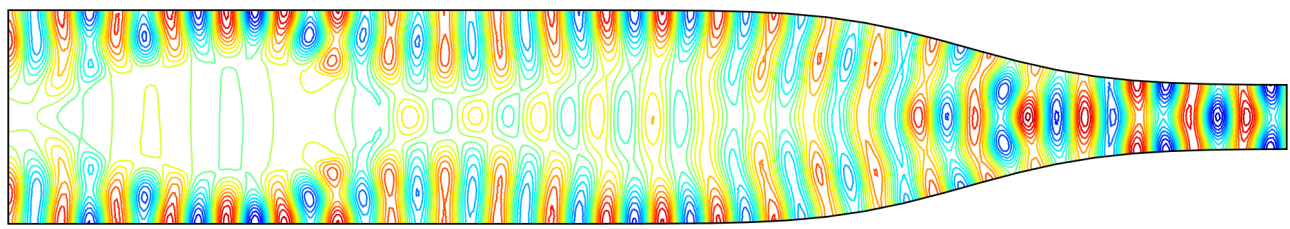

(b) contour lines of the numerical solution

Figure 11. Closed waveguide: the propagation speed $c$ is constant inside and outside of the ellipse-shaped inclusion (top); contour lines of the numerical solution of (2.1) using the CM-CG method with the functional $J_{3}$ defined by (2.26) with $t_{1}=\frac{T}{4}, t_{2}=\frac{T}{2}$ and $t_{3}=T$ (bottom).

solved in parallel with standard explicit time integration and domain decomposition methods $[5,27]$. In the presence of local mesh refinement, high-order explicit local time-stepping (LTS) methods [16] overcome the bottleneck due to an overly stringent CFL stability constraint.

The original CM-CG method is based on the minimization of the cost functional $J_{1}$ in (2.4), which guarantees convergence to the (unique) solution of the Helmholtz equation for sound-soft scattering problems when both Dirichlet and impedance boundary conditions are imposed. For any other combination of boundary conditions, however, the minimization of $J_{1}$ generally does not lead to the correct time-harmonic solution. In contrast, the cost functional $J_{3}$ in $(2.26)$ always yields the true solution at little extra computer time or memory. For scattering problems from sound-hard obstacles or inclusions, the compatibility condition (2.23)-(2.24) eliminates the spurious constant shift in the minimizer and thus even permits the use of the original cost functional $J_{1}$.

With these amendments, the CM-CG method proves an effective alternative for the numerical solution of the Helmholtz equation, be it in bounded or unbounded domains truncated by an artificial boundary. It is inherently parallel, easy to implement and particularly attractive if one has a good (time-dependent) wave equation solver at hand. The CM-CG approach developed here for the Helmholtz equation immediately generalizes to other time-harmonic vector wave equations from electromagnetics or elasticity.

Acknowledgements. We thank Martin Gander, Markus Melenk, Frédéric Nataf and Stefan Sauter for useful comments and suggestions. 
This work was partially supported by the Swiss National Science Foundation under grant SNF 200021_169243.

\section{REFERENCES}

1. Bristeau, M.-O., Glowinski, R., and PÉriaux, J.: On the numerical solution of the Helmholtz equation at large wave numbers using exact controllability methods. Application to scattering, vol. 157, pp. 399-419. Contemporary Mathematics. Amer. Math. Soc., 1994.

2. Ernst, O. G. and Gander, M. J.: Why it is Difficult to Solve Helmholtz Problems with Classical Iterative Methods, pp. 325-363. Springer Berlin Heidelberg, Berlin, Heidelberg, 2012.

3. Erlangga, Y. A., Oosterlee, C. W., and Vuik, C.: A novel multigrid based preconditioner for heterogeneous Helmholtz problems. SIAM J. Sci. Comput., 24(4), (2006), 1471-1492.

4. Engquist, B. and Ying, L.: Sweeping preconditioner for the Helmholtz equation: Moving perfectly matched layers. Multiscale Model. Simul., 9(2), (2011), 686-710.

5. Dolean, V., Jolivet, P., and Nataf, F.: An Introduction to Domain Decomposition Methods. Algorithms, Theory, and Parallel Implementation. SIAM, 2015.

6. Bollhöfer, M., Grote, M. J., and Schenk, O.: Algebraic multilevel preconditioner for the Helmholtz equation in heterogeneous media. SIAM J. Sci. Comput., 31(5), (2009), 3781-3805.

7. Bonazzoli, M., Dolean, V., Graham, I. G., Spence, E. A., and Tournier, P.-H.: A twolevel domain-decomposition preconditioner for the time-harmonic Maxwell's equations, 2017. Preprint.

8. Bristeau, M.-O., Glowinski, R., and PÉRiaux, J.: Controllability methods for the calculation of time-periodic solutions. application to scattering. J. Comput. Phys., 147(2), (1998), 265-292.

9. Lions, J.-L.: Exact controllability, stabilization and perturbations for distributed systems. SIAM J. Appl. Math., 30(2), (1988), 1-68.

10. Kř́̌žek, M., NeittaAnmäki, P., Glowinski, R., and Korotov, S.: Conjugate Gradient Algorithms and Finite Element Methods. Springer, 2004.

11. BARDos, C. and RAUCH, J.: Variational algorithms for Helmholtz equation using time evolution and artificial boundaries. Asymptotic Anal., 9(2), (1994), 101-117.

12. Koyama, D.: A controllability method with an artificial boundary condition for the exterior Helmholtz problem. Japan J. Indust. Appl. Math., 20, (2003), 117-145.

13. BABuŠKA, I. M. and SAUter, S. A.: Is the pollution effect of the FEM avoidable for the Helmholtz equation considering high wave numbers. SIAM J. Numer. Anal., 34(6), (1997), $2392-2423$.

14. Heikkola, E., Mönkölä, S., Pennanen, A., and Rossi, T.: Controllability method for acoustic scattering with spectral elements. J. Comput. Appl. Math., 204(2), (2007), 344-355.

15. Heikkola, E., Mönkölä, S., Pennanen, A., and Rossi, T.: Controllability method for the Helmholtz equation with higher-order discretizations. J. Comput. Phys., 225(2), (2007), 15531576 .

16. Grote, M. J., Mehlin, M., and Mitkova, T.: Runge-Kutta-based explicit local time-stepping methods for wave propagation. SIAM J. Sci. Comput., 37(2), (2015), 747-775.

17. LeIs, R.: Initial Boundary Value Problems in Mathematical Physics. Springer, 1st edn., 2013.

18. Jerison, D. and Kenig, C. E.: Unique continuation and absence of positive eigenvalues for Schrodinger operators. Ann. Math., 121(3), (1985), 463-488.

19. Wolff, T. H.: A property of measures in $\mathbf{R}^{N}$ and an application to unique continuation. Geom. Funct. Anal., 2(2), (1992), 225-284.

20. Graham, I. and Sauter, S.: Stability and error analysis for the Helmholtz equation with variable coefficients. Preprint.

21. Evans, L. C.: Partial Differential Equation. American Mathematical Society, 2010.

22. Lions, J. L. and Magenes, E.: Non-Homogeneous Boundary Value Problems and Applications. Springer, 2nd edn., 1972.

23. Málek, J. and Strakoš, Z.: Preconditioning and the Conjugate Gradient Method in the Context of Solving PDEs. SIAM, 2014. 
24. Bayliss, A., Gunzburger, M., and Turkel, E.: Boundary conditions for the numerical solution of elliptic equations in exterior region. SIAM J. Appl. Math., 42(2), (1982), 430-451.

25. Grote, M. J. and Keller, J. B.: On nonreflectting boundary conditions. J. Comput. Phys., 122(2), (1995), 231-243.

26. Cohen, G., Joly, P., Roberts, J. E., and Tordjman, N.: Higher order triangular finite elements with mass lumping for the wave equation. SIAM J. Numer. Anal., 38(6), (2001), 20472078.

27. Grote, M. J., Peter, D., Rietmann, M., and Schenk, O.: Newmark local time stepping on high-performance computing architectures. J. Comput. Phys., 334, (2015), 308-326.

(Marcus J. Grote) University of Basel, Basel, Switzerland

Email address, Marcus J. Grote: marcus.grote@unibas.ch

(Jet Hoe Tang) University of Basel, Basel, Switzerland

Email address, Jet Hoe Tang: jet.tang@unibas.ch 


\section{LATEST PREPRINTS}

No. Author: Title

2017-12 P. Zaspel

Algorithmic patterns for $\mathcal{H}$-matrices on many-core processors

2017-13 R. Brügger, R. Croce and H. Harbrecht

Solving a free boundary problem with non-constant coefficients

2017-14 M. Dambrine, H. Harbrecht and B. Puig

Incorporating knowledge on the measurement noise in electrical impedance tomography

2017-15 C. Bürli, H. Harbrecht, P. Odermatt, S. Sayasone and N. Chitnis Analysis of Interventions against the Liver Fluke, Opisthorchis viverrini

2017-16 D. W. Masser

Abcological anecdotes

2017-17 P. Corvaja, D. W. Masser and U. Zannier

Torsion hypersurfaces on abelian schemes and Betti coordinates

2017-18 F. Caubet, M. Dambrine and H. Harbrecht

A Newton method for the data completion problem and application to obstacle detection in Electrical Impedance Tomography

2018-01 H. Harbrecht and P. Zaspel

On the algebraic construction of sparse multilevel approximations of elliptic tensor product problems

2018-02 F. Ghiraldin and X. Lamy

Optimal Besov differentiability for entropy solutions of the eikonal equation

2018-03 H. Harbrecht and M. Schmidlin

Multilevel quadrature for elliptic problems on random domains by the coupling of FEM and BEM

2018-04 M. Bugeanu and H. Harbrecht

Parametric representation of molecular surfaces

2018-05 A. Abdulle, M. J. Grote and O. Jecker

Finite element heterogeneous multiscale method for Elastic Waves in Heterogeneous Media

2018-06 M. J. Grote and J. H. Tang

On controllability methods for the Helmholtz equation

Preprints are available under https://math.unibas.ch/research/publications 